\title{
A human mutation in STAT3 promotes type 1 diabetes through a defect in CD8+ T cell tolerance
}

Mark Anderson ( $\sim$ Mark.Anderson@ucsf.edu )

University of California at San Francisco https://orcid.org/0000-0002-3093-4758

Jeremy Warshauer

University of California at San Francisco

Julia Belk

Stanford University https://orcid.org/0000-0003-4724-6158

Alice Chan

University of California, San Francisco

Jiaxi Wang

University of California at San Francisco

Alexander Gupta

University of California at San Francisco

Dante Acenas

University of California at San Francisco

Quanming Shi

Stanford University

Yani Peng

University of California San Francisco

Jonah Phipps

University of California at San Francisco

Qizhi Tang

University of California at San Francisco https://orcid.org/0000-0001-7313-3574

James Gardner

University of California at San Francisco

Ansuman Satpathy

Stanford University School of Medicine https://orcid.org/0000-0002-5167-537X

\section{Article}

Keywords: type 1 diabetes (T1D), pathogenesis, CD8+ T cell tolerance, STAT3, mutation

Posted Date: February 25th, 2021 
DOl: https://doi.org/10.21203/rs.3.rs-257641/v1

License: (c) (1) This work is licensed under a Creative Commons Attribution 4.0 International License. Read Full License 
4 Authors: Jeremy T. Warshauer ${ }^{1,3 \dagger}$, Julia A. Belk ${ }^{5 \dagger}$, Alice Y. Chan ${ }^{7}$, Jiaxi Wang ${ }^{1}$, Alexander R. Gupta ${ }^{4}$, Dante $5 \quad$ Acenas $^{7}$, Quanming Shi ${ }^{6}$, Nikolaos Skartsis ${ }^{3}$, Yani Peng ${ }^{4}$, Jonah Phipps ${ }^{1}$, Qizhi Tang ${ }^{4}$, James Gardner ${ }^{1,4}$, Ansuman T. Satpathy ${ }^{6}$, Mark S. Anderson ${ }^{1,2,3^{*}}$

Affiliations:

$8 \quad{ }^{1}$ Diabetes Center, University of California, San Francisco, San Francisco, California 94143, USA.

$9 \quad{ }^{2}$ Department of Microbiology and Immunology, University of California San Francisco, San Francisco, California 1094143 , USA.

$11{ }^{3}$ Department of Medicine, University of California San Francisco, San Francisco, California 94143, USA.

$12{ }^{4}$ Department of Surgery, University of California, San Francisco, San Francisco, CA 94143, USA.

$13 \quad{ }^{5}$ Department of Computer Science, Stanford University, Stanford, California 94305, USA.

$14{ }^{6}$ Department of Pathology, Stanford University, Stanford, California 94305, USA.

$15 \quad{ }^{7}$ Department of Pediatrics, University of California San Francisco, San Francisco, California 94143, USA

$16 \dagger$ J.T. Warshauer and J.A. Belk contributed equally to this work.

$17{ }^{*}$ Corresponding Author

18

19 Word Count: 3,904 
Abstract:

21 Naturally occurring cases of monogenic type 1 diabetes (T1D) provide rare opportunities to establish direct

22 mechanisms that cause this complex autoimmune disease. A recently identified de novo germline gain-of-function

23 (GOF) mutation in the transcriptional regulator signal transducer and activator of transcription 3 (STAT3) was

24 shown to cause neonatal T1D at birth. To investigate the role of STAT3 hyperactivity in T1D, we engineered a

25 novel knock-in (KI) mouse incorporating this highly diabetogenic human mutation (K392R) in the STAT3 gene.

26 These mice developed accelerated diabetes with severe insulitis and insulin autoantibodies, thereby recapitulating

27 the human autoimmune diabetes phenotype. Paired T cell receptor (TCR) and transcriptome (RNA) sequencing in

28 single cells revealed that STAT3-GOF drives the proliferation and clonal expansion of highly cytotoxic effector

$29 \mathrm{CD} 8+\mathrm{T}$ cells that are resistant to terminal exhaustion. Single-cell ATAC-seq showed that these effector T cells are

30 epigenetically distinct and revealed differential chromatin architecture induced by STAT3-GOF. Analysis of islet

31 TCR clonotypes revealed an effector CD8+ T cell reacting against the known antigen IGRP, and STAT3-GOF in an

32 IGRP-reactive TCR transgenic model demonstrated that STAT3-GOF intrinsic to CD8+ T cells is sufficient to

33 accelerate diabetes onset. Taken together, these findings reveal a diabetogenic CD8+ $\mathrm{T}$ cell response that is

34 restrained in the presence of normal STAT3 activity and drives diabetes pathogenesis. 


\section{Introduction}

36 Type 1 diabetes (T1D) is caused by a breakdown of immune self-tolerance that leads to the T cell mediated destruction

37 of pancreatic $\beta$ cells and results in absolute insulin deficiency and hyperglycemia. Hallmarks of this autoimmune

38 disease include the presence of insulitis and islet autoantibodies against insulin, islet cell antigen (ICA), zinc

39 transporter 8 , and glutamic acid decarboxylase- $65^{1,2}$. Genome-wide association studies (GWAS) show T1D is a

40 polygenic disease with the majority of genetic risk attributable to human leukocyte antigen (HLA) alleles within the

41 major histocompatibility complex (MHC) region ${ }^{3}$. Numerous other susceptibility alleles (e.g., INS, CTLA4, STAT3

$42 I L 6 R)^{4,5,6,7}$ have been identified from GWAS studies and help estimate individual risk, but ultimately additional

43 functional studies are required to elucidate the cellular and molecular basis of these associations with disease.

44 Naturally occurring cases of monogenic T1D, although rare (i.e., loss-of-function mutations in Autoimmune Regulator

45 (AIRE) and FOXP3), have been most informative for understanding how central and peripheral mechanisms of

46 immune tolerance break down and for suggesting new opportunities for therapeutic interventions ${ }^{8}$.

48 Recent large scale sequencing efforts in genetics have identified naturally occurring gain-of-function (GOF) mutations

49 in the gene signal transducer and activator of transcription 3 (STAT3) as a novel cause of monogenic diabetes ${ }^{9,10}$.

50 However, it remains unclear how excessive STAT3 signaling leads to T1D. One challenge in studying STAT3 stems

51 from its ubiquitous expression and important functions in diverse cell types. Within the immune system, the Janus

kinase (JAK)-STAT signaling pathway regulates transcription in response to extracellular cues from cytokines.

53 STAT3 is a transcription factor that regulates the expression of genes associated with cell survival, proliferation,

54 activation, and differentiation ${ }^{11}$. Outside of the immune system and directly within pancreatic cells, STAT3 plays a

55 role in islet development as well as insulin secretion ${ }^{10,12,13}$. Previous work has proposed multiple conflicting

56 hypotheses regarding the relevant cell types involved in the development of diabetes caused by STAT3-GOF, such as

57 an islet-intrinsic effect or perturbing the Treg / Th17 balance within the CD4+ T cell compartment ${ }^{12,} 14,15$.

58 Observational studies have nominated a role for STAT3 in even more cell types; profiling peripheral blood in subjects

59 with T1D has demonstrated changes in STAT3-dependent pathways including increased Th17 and TFh cells in new-

60 onset T1D ${ }^{16,17}$, and IL-6 induced phosphorylation of STAT3 is significantly increased in CD4+ and CD8+ T cells of

61 patients with $\mathrm{T}^{18} \mathrm{D}^{18}$. Additional studies of human diabetogenic STAT3-GOF mutations in a physiological context are 
needed to resolve these results and establish the molecular and cellular link between STAT3 GOF mutations and T1D pathogenesis in vivo.

In this report, we generated STAT3 knock-in (KI) mice (STAT3 ${ }^{+/ K 392 R}$ ) carrying a single copy of the highly activating and diabetogenic missense mutation resulting in a substitution of arginine for lysine at amino acid 392 in the STAT3

67 DNA binding domain (K392R), which was associated with neonatal T1D in an infant at birth', ${ }^{9}$. Using this novel

68 Mendelian mouse model, we performed a battery of phenotypic, biochemical, and functional analyses of the human

69 STAT3-GOF mutation. Adoptive cell transfers and mouse genetic studies demonstrated a role for the mutation within

70 the hematopoietic system, and specifically within CD8+ T cells. We found that the mutation induces an unbridled

71 diabetogenic CD8+ T cell response, which we characterize using single cell transcriptomic, epigenetic, and $\mathrm{T}$ cell

72 repertoire profiling. We determined that while CD8+ T cell function within the islets is typically restrained by $\mathrm{T}$ cell

73 exhaustion, CD8+ STAT3 ${ }^{+/ K 392 R}$ T cells are resistant to terminal exhaustion and are maintained in a highly cytotoxic

74 state. We show that STAT3-GOF only within CD8+ T cells is sufficient to accelerate T1D in vivo, thus demonstrating

75 a cell-intrinsic role for the K392R mutation in CD8+ T cells and establishing a defect in CD8+ T cell exhaustion as a 76 contributor to T1D development.

\section{Results}

\section{Generation of a murine model of the human STAT3 K392R mutation}

80 To investigate the role of STAT3 hyperactivity in T1D, we took advantage of CRISPR/Cas9 editing to engineer a

81 novel knock-in (KI) mouse on the non-obese diabetic (NOD) background incorporating the highly diabetogenic

82 GOF human mutation (K392R) in the STAT3 gene (Fig. 1a). To confirm that the STAT3-activating behavior of this

83 mutation is conserved in mice, naïve CD4+ T cells were isolated from lymphoid organs and differentiated in vitro

84 into Th17 and Treg subsets. The STAT3 ${ }^{+K 392 R}$ T cells exhibited increased differentiation of Th17 cells relative to

85 the WT T cells while differentiation into Treg cells was reduced (Fig. 1b), which is consistent with gain-of-function

86 behavior as previously described in humans ${ }^{19,20}$.

87 
We then assessed whether STAT3 ${ }^{+/ K 392 R}$ mice exhibited the severe diabetes phenotype observed in humans. Both male and female STAT3 ${ }^{+/ K 392 R}$ mice developed diabetes more rapidly and with higher incidence than their WT

91 siblings in both sexes (Fig. 1c). To control for potential off target effects of gene editing, we used two independent

92 founder lines and confirmed this diabetic phenotype in the other founder line as well (data not shown); therefore, we

93 selected one founder line to do subsequent experiments. Young, non-diabetic mice showed normal beta cell function

94 during glucose tolerance testing (Extended Fig. 1a), and immunofluorescence of STAT3 ${ }^{+/ K 392 R}$ islets showed a

95 normal distribution of alpha and beta cells within the islets (Extended Fig. 1b) suggesting the K392R mutation did

96 not interfere with beta cell development or function as previously suggested ${ }^{12}$. Rather, diabetes onset coincided with

97 the presence of insulin autoantibodies (Extended Fig. 1c) and insulitis (Fig. 1d and Extended Fig. 1d) with a rapid

98 infiltration of B and T cells at disease-onset (Fig. 1e), supporting this mouse model's recapitulation of the

99 autoimmune diabetes phenotype observed in human patients, ${ }^{9}$,

100

101 Effector T cells drive STAT3-GOF autoimmune diabetes

102 To understand the cellular compartment driving the observed autoimmunity, immunophenotyping, genetic crosses

103 and adoptive transfer experiments were performed. Given the time course of insulitis development and diabetes

104 incidence (Fig. 1c, d), we selected 6-10 weeks as the age for studying the onset of autoimmunity in peripheral

105 lymphoid tissues and within the islets. We observed lymphoproliferation grossly by increased lymphoid organ size

106 (Extended Fig. 1e) and absolute lymphocyte cell counts within these organs of mice 6-8 weeks of age (Extended

107 Fig. 1f). Immunophenotyping of peripheral lymphocytes ex vivo by flow cytometry showed STAT3 ${ }^{+/ K 392 R}$ was

108 associated with a small increase in Th17 cells but a more marked increase in Th1 cells and an increase in Tregs (Fig.

109 2a). In addition, $\mathrm{STAT}^{+/ K 392 \mathrm{R}}$ mice had expansions of their CD8+ effector memory and CD4+ memory cellular

110 compartments (Fig. 2a).

111

112 Given the expansion of the $\mathrm{T}$ cell compartment, we asked whether the adaptive immune compartment was necessary

113 to induce diabetes by crossing STAT3 ${ }^{+/ K 392 R}$ mice to immunodeficient NOD.Rag1 ${ }^{-/ /}$mice, which lack mature B and

$114 \mathrm{~T}$ cells. STAT3 ${ }^{+/ K 392 \mathrm{R}} \mathrm{RAG1}^{-/-}$mice were completely protected from diabetes (Fig. $2 \mathbf{b}$ ) and had no insulitis, which

115 confirmed the dependence on $\mathrm{T}$ and/or B cells in driving this disease. To further refine which compartment was

116 essential in diabetes development, we investigated the role of the MHC in the STAT3 ${ }^{+/ K 392 R}$ diabetes. Similar to the 
117 HLA haplotype DR3/4 which confers the majority of T1D risk in humans, wildtype NOD mice have the high-risk

118 MHC haplotype $\mathrm{H}^{\mathrm{g}}{ }^{\mathrm{g}}$, which is essential for diabetes development. Thus, to determine whether STAT3 ${ }^{+/ K 392 \mathrm{R}}$

119 diabetes was MHC-dependent, STAT3 ${ }^{+/ K 392 R}$ mice were crossed to the NOD.H-2 ${ }^{\mathrm{b}}$ strain, which carries an alternate

120 MHC haplotype known to be protective from diabetes. NOD.H- $2^{\mathrm{b}} \mathrm{STAT}^{+/ K 392 \mathrm{R}}$ mice were completely protected

121 from diabetes (Fig. 2b) and had no insulitis upon histologic examination (data not shown) showing diabetes in

$122 \mathrm{STAT3}^{+/ K 392 \mathrm{R}}$ mice was an MHC-dependent disease.

124 The MHC-dependence suggested that diabetes in STAT3 ${ }^{+/ K 392 R}$ mice was mediated through changes either in the

125 MHC-dependent antigen expression in target pancreatic tissue or in the MHC-mediated activation of T cells

126 involved in beta-cell destruction. To establish whether the key driver of T1D was in the T cell compartment or the

127 tissue itself, bone marrow chimeras were generated by adoptively transferring WT or STAT3 ${ }^{+/ K 392 R}$ bone marrow

128 into lethally irradiated WT mice. STAT3 ${ }^{+/ K 392 R}$ bone marrow resulted in accelerated diabetes compared to WT bone

129 marrow (Fig. 2c). This result combined with the MHC-dependence showed STAT3 ${ }^{+/ K 392 R}$ induced autoimmune

130 diabetes via a T cell mediated mechanism.

132 Previous work in human subjects with STAT3-GOF disease has suggested dysfunctional Treg cells as a potential 133 cause of autoimmunity ${ }^{15}$. Therefore, we next assessed the suppressive activity of STAT3 ${ }^{+/ K 392 R}$ Treg cells in vivo.

134 Adoptive transfer of islet-antigen-specific Tregs from BDC2.5 mice, which carry a CD4-specific TCR transgene that

135 targets a pancreatic antigen derived from chromogranin A, are effective at preventing T1D in the NOD mouse

136 model $^{21,22,23}$, and we utilized this model of Treg suppression by crossing BDC2.5 mice to the STAT3 ${ }^{+/ K 392 \mathrm{R}}$ line. To

137 specifically examine the role of the STAT3 mutation in Tregs, we performed adoptive transfer of naïve WT

138 BDC2.5+ T cells into immunodeficient Rag $1^{-/-}$mice. This resulted in rapid development of diabetes that was

139 ameliorated by both WT BDC2.5+ and STAT3 ${ }^{+/ K 392 R}$ BDC2.5+ Treg cells (Extended Fig. 1g). Therefore,

$140 \mathrm{STAT3}^{+/ K 392 \mathrm{R}}$ did not significantly impair Treg function, which implicated Teff cells as key drivers of diabetes

141 development due to the STAT3 ${ }^{+/ K 392 R}$ mutation.

$143 \mathrm{~T}$ cell expansion is not due to priming by STAT3 ${ }^{+/ \mathrm{K}^{392 R}}$ APCs 
144 We next investigated whether the observed Teff expansion may be cell-intrinsic or due to improved T cell priming

145 by STAT3 ${ }^{+/ K 392 R}$ in APCs and/or the local islet environment. We performed ex vivo immunophenotyping by flow

146 cytometry of 8 week old nondiabetic STAT3 ${ }^{+/ K 392 R}$ APCs within the spleen and found STAT3 ${ }^{+/ K 392 R}$ did not

147 significantly alter population frequencies of monocytes, pre-DCs or DCs (Fig. 3a). We next immunophenotyped

148 APCs directly within the islets using mass cytometry, and observed the expression of classical molecules needed for

149 antigen presentation to T cells, MHC class II and CD86, also appeared undisturbed in 8-10 week old nondiabetic

150 mice (Fig. 3b). We then performed transcriptional profiling of the infiltrating immune cells using scRNA-seq of

151 CD45+ lymphocytes isolated from islets of 8-10 week old nondiabetic mice. We obtained high quality scRNA

152 profiles for 20,361 high quality single cells from STAT3 ${ }^{+/ K 392 R}$ and WT mice. Cells were clustered and then

153 visualized using Seurat (Extended Fig. 2a), followed by a reclustering of only the CD11c+ APCs (Fig. 3c) for

154 further analysis. Expression of canonical immune cell markers were computed and used to uniquely identify the six

155 cell populations represented by each cluster, which included pDCs, cDCs, macrophages and monocytes (Extended

156 Fig. 2b). Overall, cluster population frequencies were similar between STAT3 $3^{+/ K 392 R}$ and WT cells (Fig. 3d),

157 consistent with our flow cytometry data (Fig. 3a). Corroborating our mass cytometry data (Fig. 3b), expression of

158 antigen presenting genes $C d 86$ and MHC-II (H2-Abl) appeared comparable (Fig. 3e) among the different APC

159 populations. Altogether, these results supported phenotypic similarity between STAT3 ${ }^{+/ K 392 R}$ and WT APCs.

161 Finally, we performed a functional experiment to test whether STAT3 ${ }^{+/ K 392 R}$ in APCs and/or the local islet

162 environment was priming T cells and driving their increased proliferation. Naïve CD4+ BDC2.5Tg+ cells were

163 isolated by FACS from non-diabetic BDC2.5Tg+ donors, labeled with CTV to track cellular activation and

164 proliferation, and adoptively transferred into STAT3 ${ }^{+/ K 392 R}$ or WT hosts. Proliferation was evaluated 3.5 days after

165 cell transfer by FACS analysis of the CTV dye dilution in the pLNs and iLNs (control) of recipient mice, and there

166 was no evidence to support the local islet environment as a driver of $\mathrm{T}$ cell priming and activation (Fig. 3f). Overall,

167 the lack of evidence to support an effect of STAT3 ${ }^{+/ K 392 R}$ on the APC compartment suggested the diabetogenic effect

168 of STAT3 ${ }^{+/ K 392 R}$ was intrinsic to the Teff cells rather than their surrounding environment. 
171 To better understand the molecular mechanisms of STAT3 ${ }^{+/ K 392 R}$ within Teff cells, we performed paired

172 transcriptome and T cell receptor sequencing in single cells (scRNA / TCR-seq) from CD45+ lymphocytes isolated

173 from islets of the 8-10 week old nondiabetic mice used in the earlier scRNA-seq experiment (Extended Fig. 2a). A

174 total of 8,725 CD3+ T cells were reclustered and used for further analysis (Fig. 4a). Differentially expressed marker

175 genes for each cluster were computed (Extended Fig. 2c) along with canonical marker gene expression (Extended

176 Fig. 2d, e) and used to uniquely identify the cell populations represented by each T cell cluster. Comparisons of the

$177 \mathrm{STAT3}^{+/ K 392 \mathrm{R}}$ vs WT clusters showed a marked expansion of effector CD8+ T cells and a corresponding decrease in

178 naïve CD8+ T cells (Fig. 4a). Notably, no increase in Treg or Th17 cell populations in these islet infiltrates was

179 observed. Differential gene expression comparing the STAT3 ${ }^{+/ K 392 R}$ vs WT CD8+ T cells showed STAT3 ${ }^{+/ K 392 R}$

180 drove a highly cytotoxic CD8+ T cell phenotype with upregulation of genes involved in chemotaxis (e.g., Ccl4 and

$181 C c l 5)$ and cytotoxicity (e.g., Gzma, Gzmb, Gzmk) (Fig. 4b). To confirm these findings at the protein level, we

182 performed flow cytometry and found that STAT3 ${ }^{+/ K 392 R} \mathrm{CD} 8+\mathrm{T}$ cells isolated from the pancreatic lymph node

183 showed increased Ccl5 expression relative to WT (Fig. 4d), and CD8+ T cells from the spleen showed increased

184 Granzyme A and Granzyme B protein expression (Extended Fig. 2f).

186 To provide deeper characterization of the expanded STAT3 ${ }^{+/ K 392 R} \mathrm{CD} 8+\mathrm{T}$ cells, a total of 2,034 CD8+ T cells were 187 reclustered for further analysis (Fig. 4d). Clustered cell populations were identified by their expression of canonical 188 CD8 markers (Fig. 4e), in addition to using module scores to distinguish terminally exhausted T cells (Cd101,

$189 C d 200 r 2, C d 7, C d 200 r 1, I l 10)$ and transitory T cells (Cx3crl, Klrg1, Il2ra, Ill8rap, Slpr5) (Fig. 4f), as previously 190 described $^{24}$. Focusing on the expanded non-naïve cell populations, STAT3 ${ }^{+/ K 392 R}$ cells exhibited a bias towards the 191 transitory (vs terminally) exhausted CD8+ T cell phenotype (Fig. 4g) with STAT3 ${ }^{+/ K 392 R}$ transitory:terminally 192 exhausted ratio 2.5 times that of WT. As the gene signatures of the effector and transitory clusters (Fig. 4h) closely 193 matched that of the differential gene expression seen across CD8 T cells in aggregate (Fig. 4b), it suggested the 194 phenotypic difference in CD8 T cells caused by STAT3 ${ }^{+/ K 392 R}$ was driven by an increased proportion of transitory 195 cells with a cytotoxic gene expression profile, while WT cells preferentially exhibited a terminally exhausted 196 phenotype.

198 STAT3-GOF epigenetically regulates chemotactic and cytotoxic genes in effector CD8+ T cells 
199 In parallel to our single cell transcriptomic approach, we also used single cell epigenetics to examine the chromatin

200 state in infiltrating immune cells in the islets. We performed scATAC-seq on CD45+ infiltrating islet cells of 8-10

201 week old nondiabetic mice similar to those used in the scRNA-seq studies and obtained high-quality ATAC-seq

202 profiles from 17,466 single cells with a median number of 5,610 fragments per cell and a median enrichment of Tn5

203 insertions in transcription start sites for 17.11 (Extended Fig. 3). To identify cell-types and determine a

204 correspondence between the scRNA-seq clusters and the scATAC-seq clusters we used gene scores, which are

205 computed by aggregating the ATAC-seq signal across the gene body and promoter in each cell (Fig. 5a). By

206 analyzing the corresponding clusters, we were able to link our gene expression findings with epigenetic changes in

207 the same cell types. There were 197 peaks with significantly increased chromatin accessibility whose nearest gene

208 was one of the 129 significantly upregulated genes ( $\log \mathrm{FC} \geq 0.25$ and padj $\leq 0.05$, Fig. 5b, c). Key molecules

209 characteristic of cytotoxic CD8+ T cells, including Ccl5, Klrgl, and Gzma, had multiple nearby significant peaks

210 (Fig. 5b), indicating epigenetic regulation of the effector T cell gene expression program induced in STAT3 ${ }^{+/ K 392 \mathrm{R}}$

211 compared to WT mice. We next sought to determine which transcription factors might be responsible for the

212 observed epigenetic remodeling in the CD8+ T cell compartment. Comparing transcription factor motif accessibility

213 between STAT3 $^{+/ K 392 R}$ and WT CD8+ T cells using chromvar ${ }^{25}$ identified 406 motifs with significantly different

214 activity (FDR $\leq 0.1, \mid$ mean difference $\mid \geq 0.01$ ). Eomes and $T b x 21$ were among the significantly more accessible

215 transcription factors and have well established roles in effector T cell development and function ${ }^{26}$ (Fig. 5d, e).

216 Transcription factors involved in programming tissue residency in CD8+ T cells were among the most significantly

217 different, in particular the increased accessibility of Runx $3^{27}$ and decreased accessibility of Klf2 ${ }^{28}$ (Fig. 5d, e). In

218 sum, STAT3 ${ }^{+/ K 392 R}$ leads to increased activity of TFs involved in tissue infiltration and cytotoxic gene expression

219 programs in CD8+ effector T cells.

\section{STAT3-GOF in diabetogenic CD8+ $T$ cells are sufficient to accelerate T1D}

222 Finally, we sought to establish whether these STAT3 ${ }^{+/ K 392 R}$ effector CD8+ T cells were directly responsible for the

223 autoimmune diabetic phenotype. We analyzed the TCR repertoire data obtained from the scRNA / TCR-seq

224 experiment to characterize the specificity of the infiltrating CD8+ T cells and determine whether they were indeed

225 directly diabetogenic. We recovered high-confidence TCR sequences for $91 \%$ of T cells (Extended Fig. 4a). We

226 used the Gini index to quantify the extent of TCR clonal expansion within each cluster and found the STAT3 $3^{+/ K 392 R}$ 
effector CD8+ T cell cluster exhibited increased clonal expansion relative to WT (Extended Fig. 4b) suggesting that these cells were recognizing and proliferating in response to islet antigens. We then analyzed the top 20 most abundant CD8+ TCR clones and identified Clone 6158, which was present in both STAT3 ${ }^{+/ K 392 R}$ and WT mice (Fig. 6a). This specific clone contained CDR3 sequences nearly identical to those found in the TCR-8.3 (Fig. 6b), a CD8-

231 restricted TCR specific for the islet-specific glucose-6 phosphatase-related protein [IGRP] antigen that is known to

232 be diabetogenic in NOD mice $(8.3 \mathrm{Tg}+)^{29}$. Since this clone was shared between the two genotypes, we crossed the

$2338.3 \mathrm{Tg}+$ mouse with the $\mathrm{STAT3}^{+/ \mathrm{K} 392 \mathrm{R}}$ line and assessed diabetes incidence to isolate whether the underlying

234 transcriptional and epigenetic changes were key drivers underlying the STAT3 ${ }^{+/ K 392 R}$ diabetogenic effect. Consistent 235 with a STAT3-GOF intrinsic effect on CD8+ T cells, STAT3 ${ }^{+/ K 392 \mathrm{R}} 8.3 \mathrm{Tg}+$ mice became rapidly diabetic and with a 236 much higher diabetes incidence than WT 8.3Tg+ (Fig. 6c).

238 To then test whether STAT3-GOF in diabetogenic CD8+ T cells was sufficient to accelerate T1D, we used an 239 established adoptive transfer model in which polyclonal CD4+ T cells are transferred with naïve 8.3-TCR transgenic $240 \mathrm{CD} 8+\mathrm{T}$ cells and start inducing diabetes within a month of transfer into NOD.SCID recepients ${ }^{30,31,32}$. NOD.SCID 241 mice adoptively transferred with polyclonal WT CD4+ T cells and naïve CD8+ T cells from STAT3 ${ }^{+/ K 392 R} 8.3 \mathrm{Tg}+$ 242 mice experienced significantly accelerated diabetes onset compared to mice who instead received their naïve CD8+ 243 T cells from WT 8.3Tg+ mice (Fig. 6d). As no other immune populations were present in the recipient NOD.SCID 244 mice, this experiment established STAT3-GOF in diabetogenic CD8+ T cells is sufficient to drive the autoimmune 245 diabetic phenotype. We also performed adoptive transfer experiments to understand whether CD4+ cells might also 246 be playing a key role in STAT3-GOF diabetes and did not find evidence to support this. First, naïve CD8+ cells 247 from WT $8.3 \mathrm{Tg}+$ mice transferred with polyclonal CD4+ cells from either STAT3 ${ }^{+/ K 392 \mathrm{R}}$ or WT mice into 248 NOD.SCID mice did not cause a noticeable difference in diabetes incidence, which suggested the diabetogenic 249 effect of STAT3 ${ }^{+/ K 392 R}$ was likely intrinsic to its role in CD8+ T cells rather than an indirect result of CD4 help to 250 the CD8+ population (Extended Fig. 4c). Second, the adoptive transfer of either WT BDC2.5+ or STAT3 $3^{+/ K 392 R}$ 251 BDC2.5+ CD4+ Teff cells into NOD.Rag ${ }^{-/-}$mice did not result in differing diabetes incidences, suggesting CD4+ 252 cells alone were insufficient drivers of the accelerated diabetic phenotype (Extended Fig. 4d). Altogether, these 253 findings showed effector CD8+ cells were the key drivers of STAT3-GOF diabetes, and in combination with our 
254 molecular data, established a direct mechanism used by STAT3 to promote T1D through a defect in CD8+ T cell 255 tolerance.

\section{Discussion}

258 In sum, we generated a novel Mendelian model of monogenic autoimmune diabetes based on a human STAT3-GOF

259 mutation, identified how it caused an unbridled autoreactive effector CD8+ T cell response at the epigenetic and

260 transcriptomic levels, and confirmed that these findings are sufficient to accelerate T1D in vivo, thus establishing a

261 novel direct pathway involved in T1D pathogenesis. Our findings are surprising given prior assumptions from ex

262 vivo and in vitro studies were STAT3-GOF caused T1D via Th17/Treg imbalance ${ }^{14,15}$ or an islet intrinsic defect ${ }^{12}$,

263 and highlights the value of in vivo study of human mutations to uncover mechanisms underlying diseases.

264 However, our findings are also consistent with STAT3's known role in driving formation of memory CD8+ T

265 cells $^{33}$, which have a highly proliferative potential and long-term survival, consistent with the CD8+ T cell

266 expansions we observed in our STAT3-GOF mouse model in the secondary lymphoid organs and islets. STAT3 is

267 required to sustain the expression of $\mathrm{EOMES}^{34}$, another key transcription factor involved in the effector to memory

268 cell transition and which we observed had more motif activity in our ATAC-seq data. Because the mutation we

269 studied is in the DNA binding domain, we speculate STAT3-GOF is inducing an opening of chromatin regions

270 within activated CD8+ T cells that are associated with chemotaxis (e.g., Ccl4 and Ccl5), cytotoxicity (e.g., Gzma,

271 Gzmb, Gzmk), and memory homeostasis (e.g., Tbx21 and Eomes), which leads to a more diabetogenic CD8+ T cell

272 population.

274 Additionally, our findings suggest STAT3 ${ }^{+/ K 392 R}$ causes excessive CD8 T cell effector activity by impeding the 275 development of terminal exhaustion and instead retaining CD8 effector cells in a highly cytotoxic (and typically 276 transitory) phenotype. It has been shown CD8 exhaustion follows a spectrum ${ }^{35}$, with different exhausted CD8 T cell

277 subsets retaining different levels of effector functions ${ }^{36,37,38}$. The transitory CD8 population, similar to Tex ${ }^{\text {int }}$

278 previously described ${ }^{35}$, carries more proliferative capacity and effector-like function (e.g., upregulation of genes

279 such as $T b x 21$ and $G z m b$ ) compared to terminally exhausted CD8 cells that are less functional ${ }^{24}$. This aligns with our

280 findings of an expanded CD8 T cell compartment containing a similar cytotoxic profile, and which subsequently

281 induces acceleration of diabetes onset. The transitory population also expands in response to PD-1 pathway 
282 blockade and is thought to play a critical role in therapeutic response to checkpoint blockade used in cancer

283 immunotherapy ${ }^{24}, 35$. Separately, it has been shown that $\mathrm{T}$ cell exhaustion is important in determining autoimmune

284 disease outcomes ${ }^{39}$ and PD-1 deficient NOD mice develop accelerated diabetes ${ }^{40,41}$. Our findings help bridge these

285 important sets of knowledge by expanding the role of transitory exhausted T cells to autoimmune disease and T1D.

286 This is consistent with recent observations from studying T1D progression in humans showing individuals with a

287 higher activated transitional memory CD8 phenotype tended to progress more rapidly to T1D, while individuals

288 with a more terminally exhausted CD8 phenotype had a slower rate of T1D progression ${ }^{42}$. Our results also align with

289 the positive associations between exhausted CD8+ T cells and T1D immunotherapy treatment response during

290 landmark T1D prevention and reversal trials using the anti-CD3 monoclonal antibody teplizumab ${ }^{43,44}$. This

291 underscores how using a mouse model rooted in a human mutation can provide cellular and molecular insights into

292 the determinants of response in human T1D clinical trials. In addition, this work may help further our understanding

293 of how unintended autoimmune toxicities, such as T1D, result from cancer immunotherapies and how we might

294 develop strategies to prevent them ${ }^{45}$. Altogether, our new model has allowed us to determine how this transitory

295 state may be a key tolerance pathway that keeps T1D in check.

297 Historically, T1D development has been studied in the context of central tolerance mechanisms (via the study of

298 human AIRE mutations) and peripheral suppressor mechanisms (via the study of human FOXP3 mutations).

299 Therefore, our findings fill an important void necessary for understanding this complex autoimmune disease by

300 establishing that CD8+ T cell tolerance also plays a key role in T1D immunopathogenesis ${ }^{46,47,48}$. Our studies

301 support an important protective role for CD8 T cell exhaustion in T1D and suggest modulating CD8 T cell function

302 may be a therapeutic opportunity for the prevention and treatment of human T1D. 
Figure 1. STAT3 ${ }^{+/ K 392 R}$ mutant mice recapitulate the human type 1 diabetes phenotype $\mid$ a, STAT3 ${ }^{\mathrm{K} 392 \mathrm{R}}$ mutation located in the DNA binding domain was inserted into WT (i.e., NOD) mice using CRISPR/Cas9 and confirmed by Sanger sequencing $\mathbf{b}$, In vitro CD4 differentiation of naïve CD4+ T cells into Th17 and Treg subsets. c, Diabetes incidence curves (Females: WT, $n=93$; STAT3 ${ }^{+K 392 R}, n=125$; Males: WT, $n=19$; STAT3 ${ }^{+/ K 392 R,} n=68$ ) d, Time course of insulitis in WT and STAT3 $3^{+/ K 392 R}$ mice with and without diabetes $(n=3-11$ mice per gender per time point) $\mathbf{e}$, viSNE plots of islets using mass cytometry compare immune and endocrine cellular compositions between a WT mouse without diabetes and littermate STAT3 ${ }^{+/ K 392 R}$ mouse with recently-diagnosed diabetes to highlight the presence of immune infiltration with STAT3 ${ }^{+/ K 392 R}$ diabetes. Results representative of viSNE plots from 8-14 week old non-diabetic WT $(n=6)$ and diabetic STAT3 ${ }^{+/ K 392 R}(n=4)$ mice. Error bars indicate mean $+/-$ SD. ns $=$ non-

a

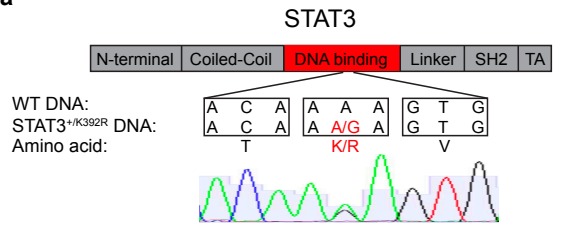

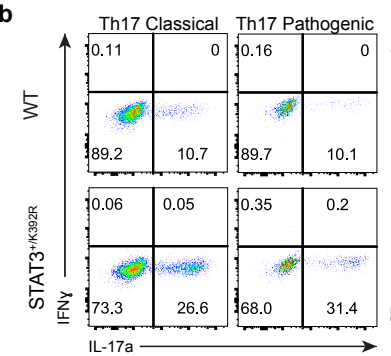
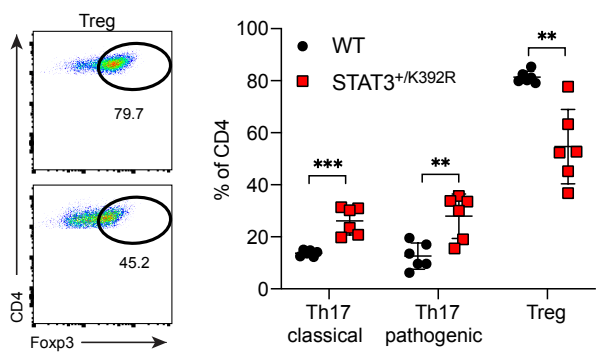

c
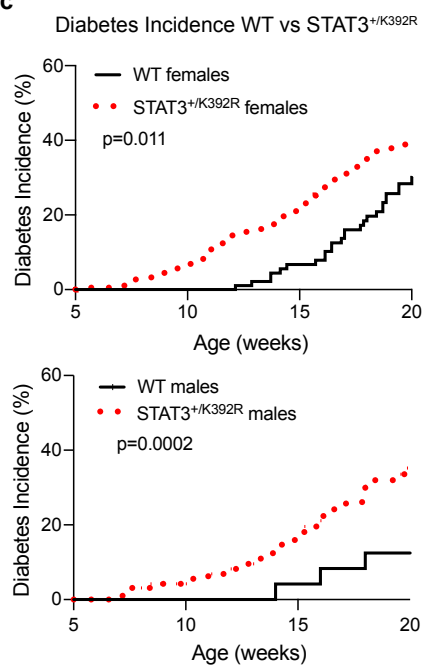

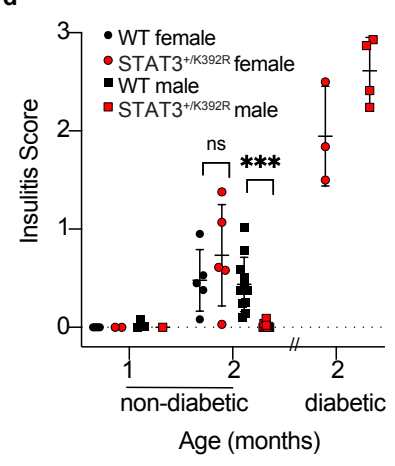

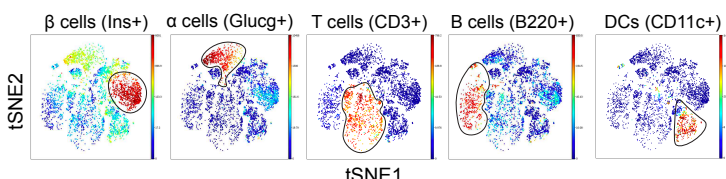

tSNE1

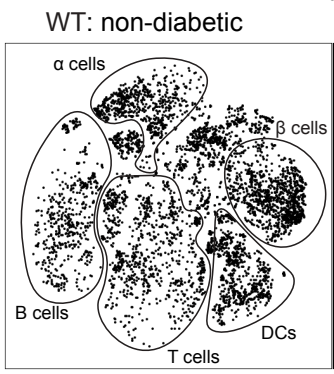

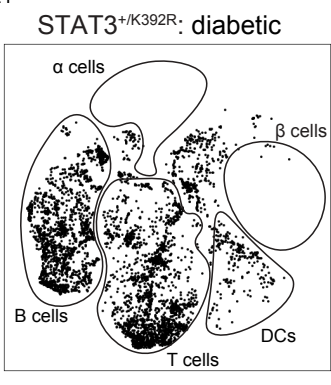


Figure 2. STAT3 ${ }^{+/ K 392 R}$ effector $\mathbf{T}$ cell compartment $\mid$ a, Immunophenotyping of splenocytes ex vivo in 8 week old male mice ( $\mathrm{n}=4$ per group) $\mathbf{b}$, Diabetes incidence in STAT3 ${ }^{+/ K 392 R} \mathrm{RAG1} 1^{-/}$mice (B- and T-cell dependence) and $\mathrm{STAT3}^{+/ K 392 \mathrm{R}} \mathrm{H}^{\mathrm{b} / \mathrm{b}}$ mice (MHC-dependence) (STAT3 ${ }^{+/ K 392 \mathrm{R}}$ females, $\mathrm{n}=132$; STAT3 $^{+/ \mathrm{K} 392 \mathrm{R}}$ males, $\mathrm{n}=70$; $\mathrm{STAT3}^{+/ K 392 \mathrm{R}} \mathrm{RAG}^{-/-}, \mathrm{n}=9$; $\mathrm{STAT}^{+/ \mathrm{K} 392 \mathrm{R}} \mathrm{H} 2^{\mathrm{b} / \mathrm{b}}, \mathrm{n}=20$ ) $\mathbf{c}$, Experimental design and subsequent diabetes incidence in bone marrow chimeras - lethally irradiated WT recipients adoptively transferred with bone marrow from WT $(\mathrm{n}=18)$ or $\mathrm{STAT3}^{+/ K 392 R}(\mathrm{n}=17)$ mice. Error bars indicate mean $+/$ - SD. $\mathrm{ns}=$ non-significant $(\mathrm{P}>0.05), * \mathrm{P} \leq 0.05, * *$ $\mathrm{P} \leq 0.01, * * * \mathrm{P} \leq 0.001$.
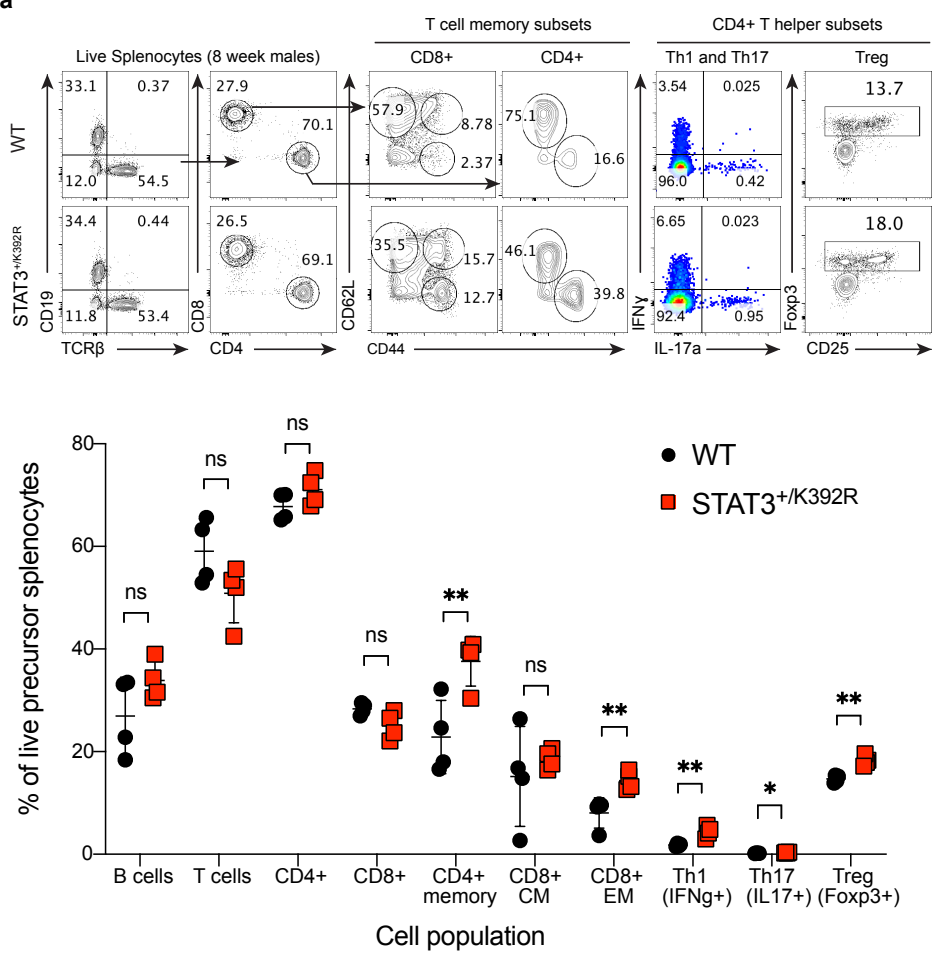
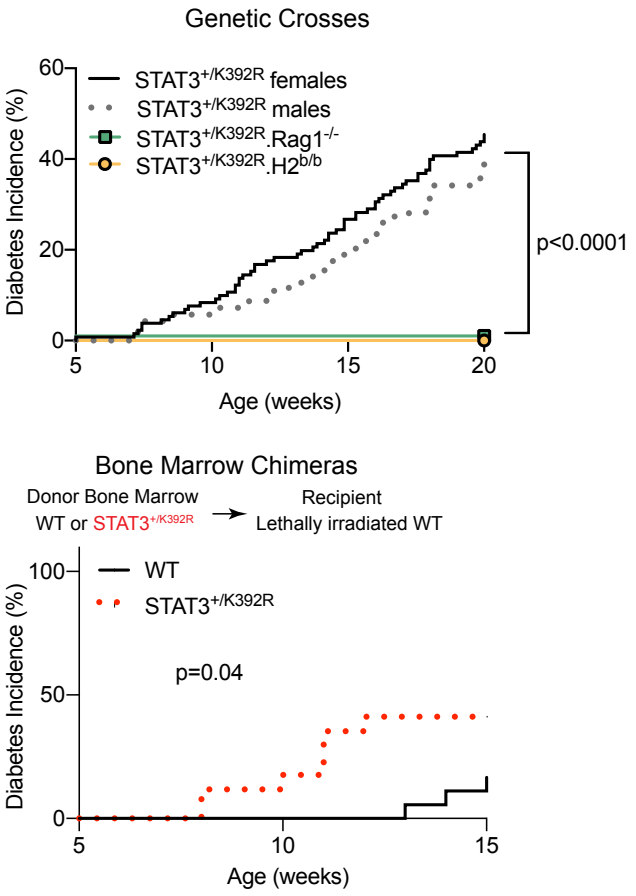
Figure 3. STAT3 ${ }^{+/ K 392 R}$ APC Phenotype and T-cell priming by the local islet environment is unchanged | a, Flow cytometry gating (values shown are frequency relative to parent gate) and population frequencies of APC subpopulations from splenocytes isolated ex vivo in non-diabetic 8 week old male mice ( $\mathrm{n}=4$ per group) b, Representative histograms for MHCII and CD86 protein expression on conventional DCs (CD11 c+MHC II + ) and classical monocytes $(\mathrm{CD} 11 \mathrm{~b}+\mathrm{Ly} 6 \mathrm{c}+)$ in the islets of non-diabetic 8-10 week old mice using mass cytometry. Performed in 3-4 mice per group c, UMAP projection of reclustered antigen-presenting myeloid cells (CD11c+ cells) from islet immune infiltrate used in scRNA-seq experiment (see Extended Fig. 2a) d, UMAP projection showing distribution of STAT3 ${ }^{+/ K 392 R}$ and WT cell transcriptomes e, Violin plots showing gene expression profile of MHCII and CD86 in APC myeloid clusters f, CTV profile among BDC2.5 CD4+ T cells in pancreatic and inguinal (control) lymph nodes (pLN and iLN, respectively) 3.5 days after adoptive transfer into STAT3 ${ }^{+/ K 392 R}$ and WT recipients. Cell proliferation was evaluated by FACS analysis of the CTV dilution in the pLNs and iLNs of recipient mice and results are expressed as the percentage of proliferating cells within the recovered CTV-labeled BDC2.5 CD4+ T cells. Representative of three sets of STAT3 ${ }^{+/ K 392 R} /$ WT recipient mice. Error bars indicate mean +/- SD. ns $=$ non-significant $(\mathrm{P}>0.05)$.

a

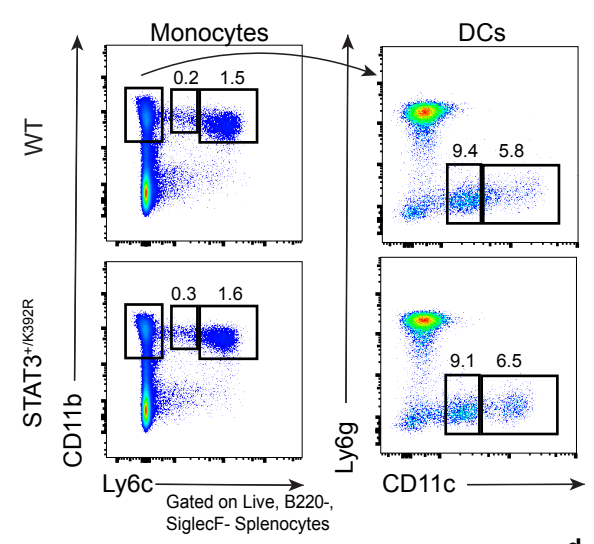

b

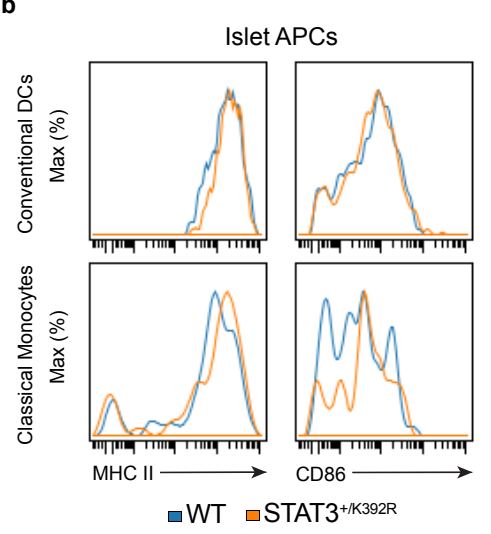

C

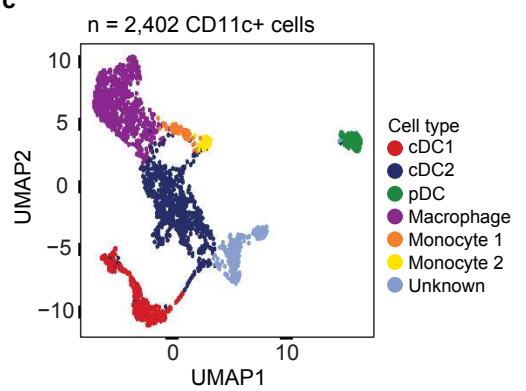

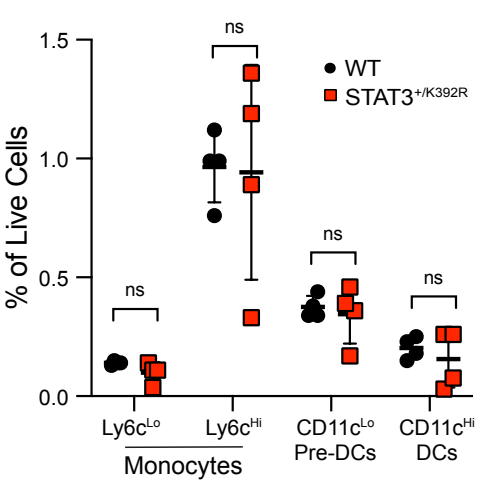

e

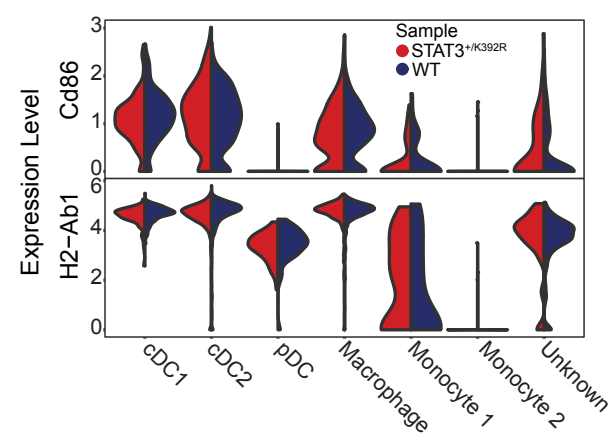

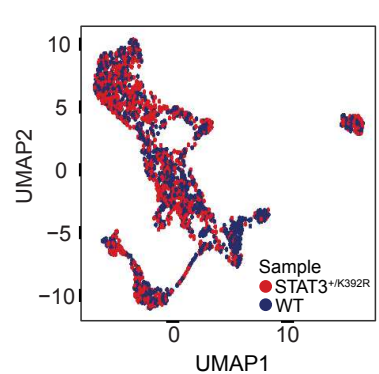

f
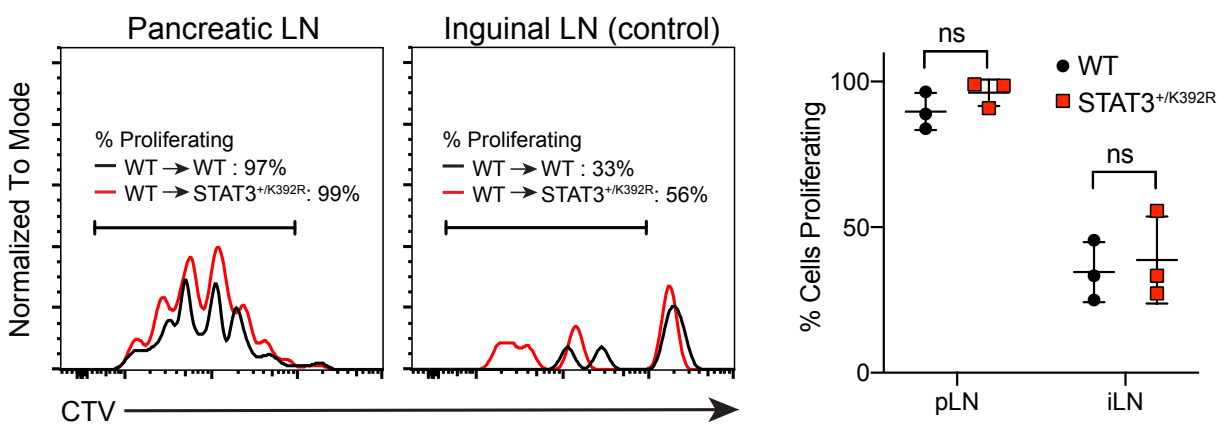
Figure 4. STAT3 ${ }^{+/ K 392 R}$ drives expansion of the CD8+ effector compartment with upregulation of cytokine and scRNA-seq experiment (see Extended Fig. 2a) with associated cell counts per cluster and comparison of cluster frequencies between the two genotypes (STAT3 ${ }^{+/ K 392 R}$ vs WT) b. Volcano plot showing STAT3 $3^{+/ K 392 R}$ vs WT differential gene expression in CD8+ clusters. Log $N$ fold cutoff 0.25 used. Genes of interest are labeled. c, Flow cytometry of $\mathrm{Ccl} 5$ protein expression in $\mathrm{CD} 8+$ memory $\mathrm{T}$ cells from the pancreatic lymph node in nondiabetic males at 14 weeks ( $\mathrm{n}=3$ per group). Error bars indicate mean $+/$ - $\mathrm{SD}, \mathrm{ns}=$ non-significant $(\mathrm{P}>0.05),{ }^{*} \mathrm{P} \leq 0.05$. d, UMAP projection of reclustered CD8+ T cells e-f, Marker gene or module score expression in CD8+ T cell clusters. In $\mathbf{f}$, genes used to calculate module scores for terminally exhausted T cells were Cd101, Cd200r2, Cd7, Cd200r1, Il10 and for transitory T cells were $C x 3 \mathrm{crl}$, Klrgl, Il2ra, Ill8rap, Slpr5, as previously described ${ }^{24} \mathbf{g}$, Relative population frequencies among non-naïve CD8+ cell clusters $\mathbf{h}$, Volcano plot showing differential gene expression in CD8+ effector and transitory cell clusters relative to all CD8+ cells.
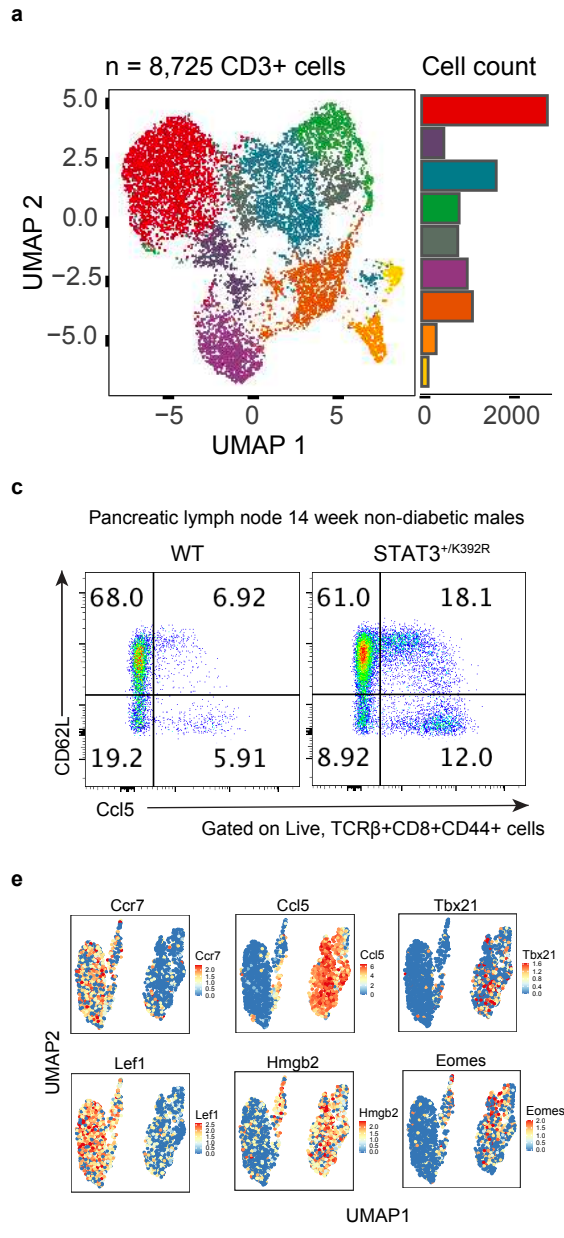
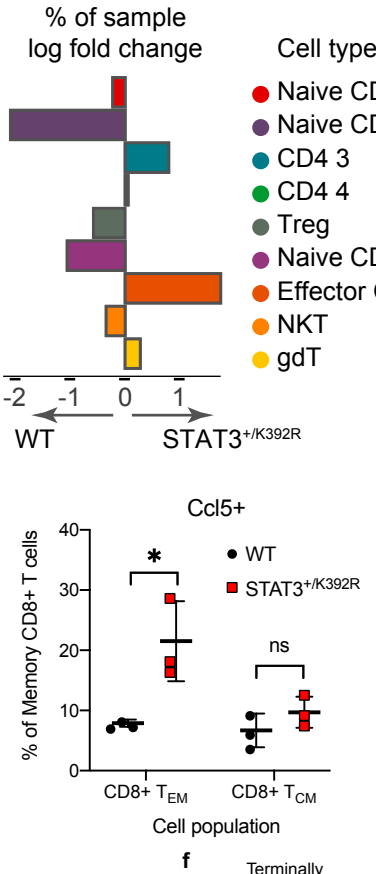

$\mathbf{f}$
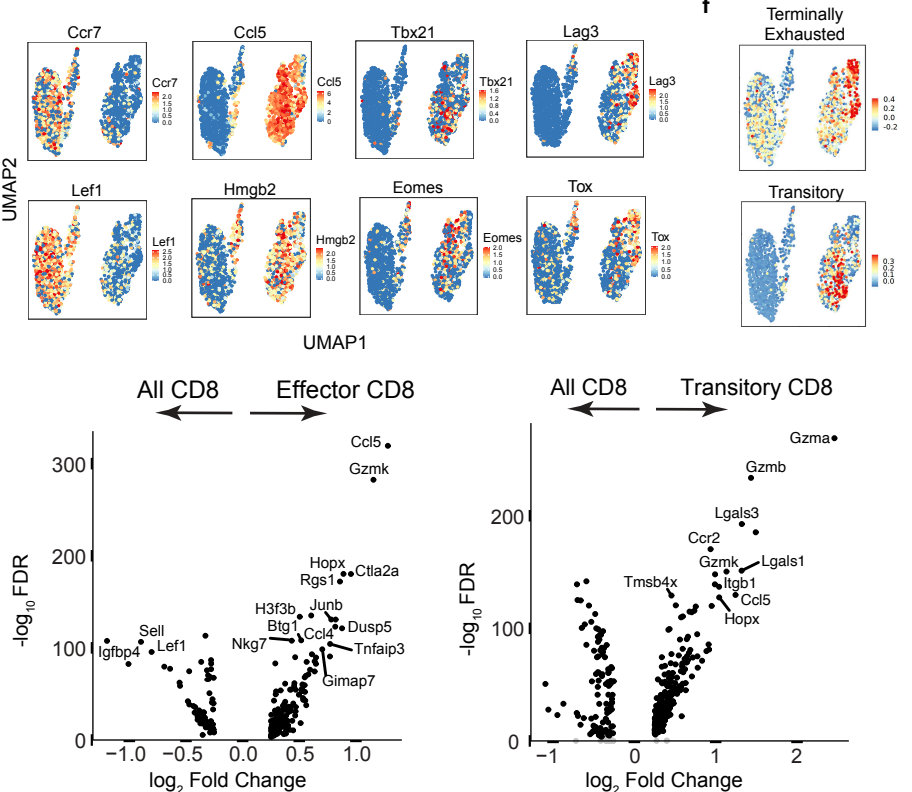

Cell type

- Naive CD4 1

- Naive CD4 2

- CD4 3

- CD4 4

- Treg

- Naive CD8

- Effector CD8

- NKT

gdT

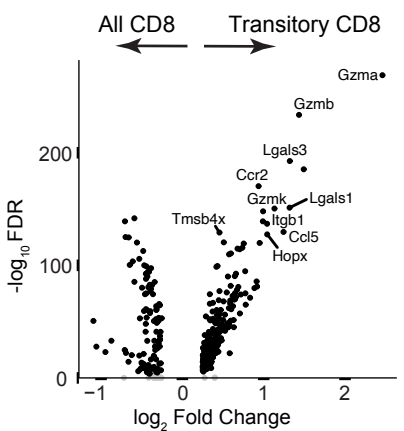

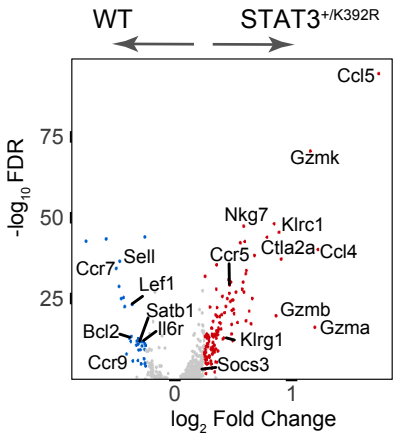

d
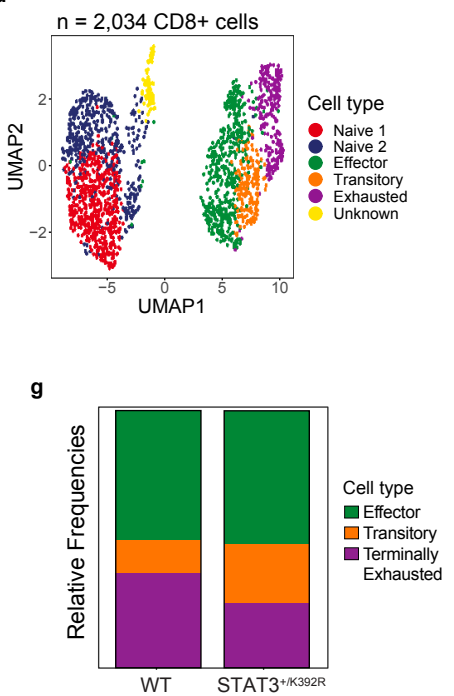
Figure 5. STAT3 ${ }^{+/ K 392 R}$ increases chromatin accessibility within cytotoxic CD8+ loci resulting an epigenetically distinct state $\mid$ a, UMAP projection of scATAC-seq profiles of T cells (CD3+) subclustered from CD45+ islet infiltrates of 8-10 week old male non-diabetic mice, WT $\left(n=3\right.$, pooled) vs STAT3 ${ }^{+/ K 392 R}(n=3$, pooled) b, Normalized pseudobulk ATAC-seq tracks of CD8+ T cell clusters split by genotype around genes identified as upregulated in corresponding scRNA-seq CD8+ T cell clusters. Peaks significantly upregulated (from c) are shown in red c, Volcano plot showing differential peaks whose nearest gene is present in the significantly upregulated genes (Fig. 4b) between CD8+ T cell clusters (STAT3 ${ }^{+/ K 392 R}$ vs WT) d, Volcano plot showing differential transcription factor motifs between CD8+ T cell clusters $\left(\mathrm{STAT}^{+/ \mathrm{K}^{392 R}}\right.$ vs WT) e, Transcription factor motif activity for $\mathrm{CD} 8+\mathrm{T}$ cell clusters split by genotype. Motif sequence shown above each violin plot.
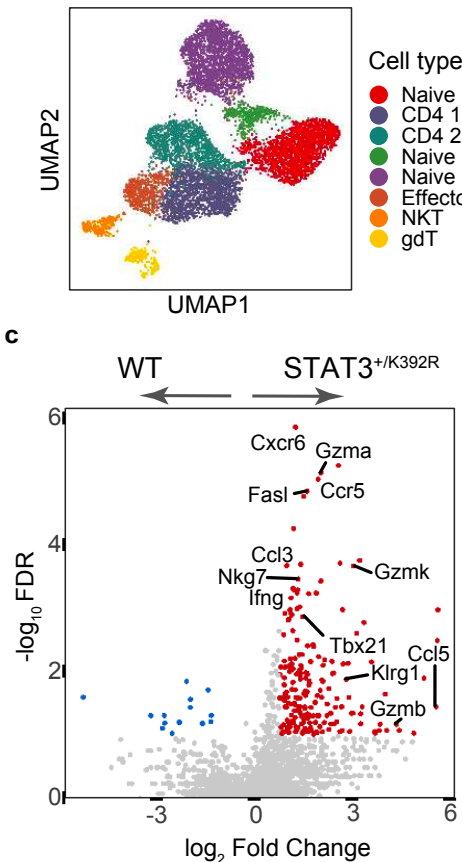
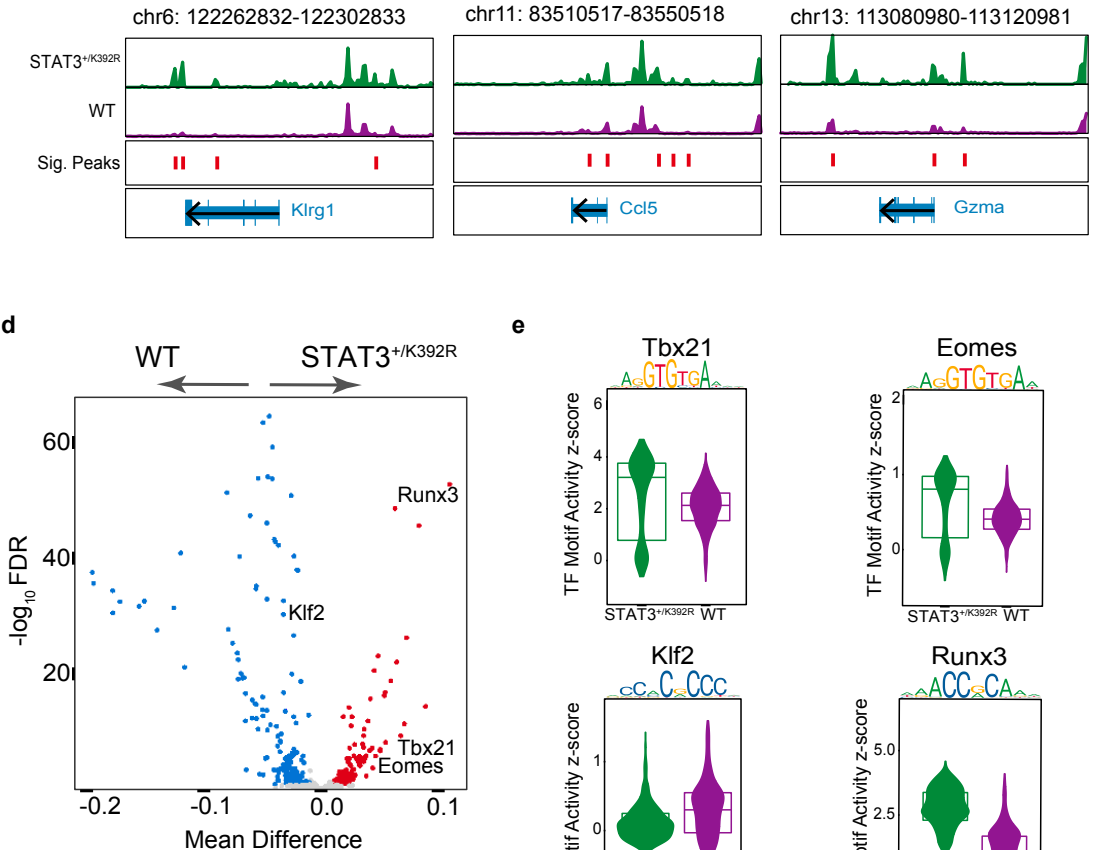

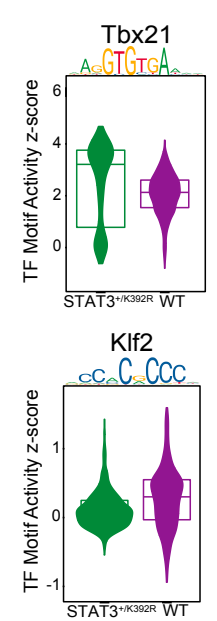

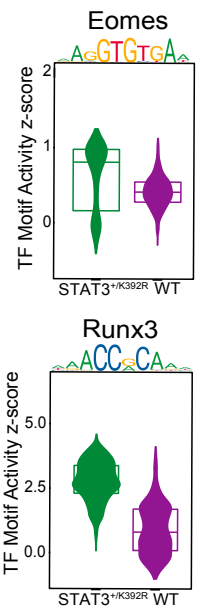


Figure 6. STAT3 ${ }^{+/ K^{392 R}}$ drives clonal expansion of diabetogenic CD8+ $\mathbf{T}$ cells $\mid \mathbf{a}$, Top 20 clones by count in each sample b, Top 10 specific clones with corresponded CDR3 sequences displayed in table. The CDR3 sequence for Clone 6158 in red is nearly identical to that of the CD8-restricted TCR specific for islet specific antigen IGRP (TCR-8.3, sequence shown below table). The only difference between Clone 6158 and TCR-8.3 is a serine, denoted in blue, replaces an alanine of the TCR beta chain $\mathbf{c}$, Diabetes incidence of $8.3 \mathrm{Tg}+$ (IGRP-specific TCR) mice with $(\mathrm{n}=7)$ and without $(\mathrm{n}=11) \mathrm{STAT}^{+/ K 392 \mathrm{R}}$ confirms increased diabetogenicity d, Diabetes incidence after adoptive transfer of polyclonal WT CD4+ T cells with naïve CD8+ T cells from $8.3 \mathrm{Tg}+$ mice with $(\mathrm{n}=5)$ and without $\mathrm{STAT3}^{+/ K 392 \mathrm{R}}(\mathrm{n}=10)$ into NOD.SCID mice. Results are pooled from 2 independent cohorts.

a
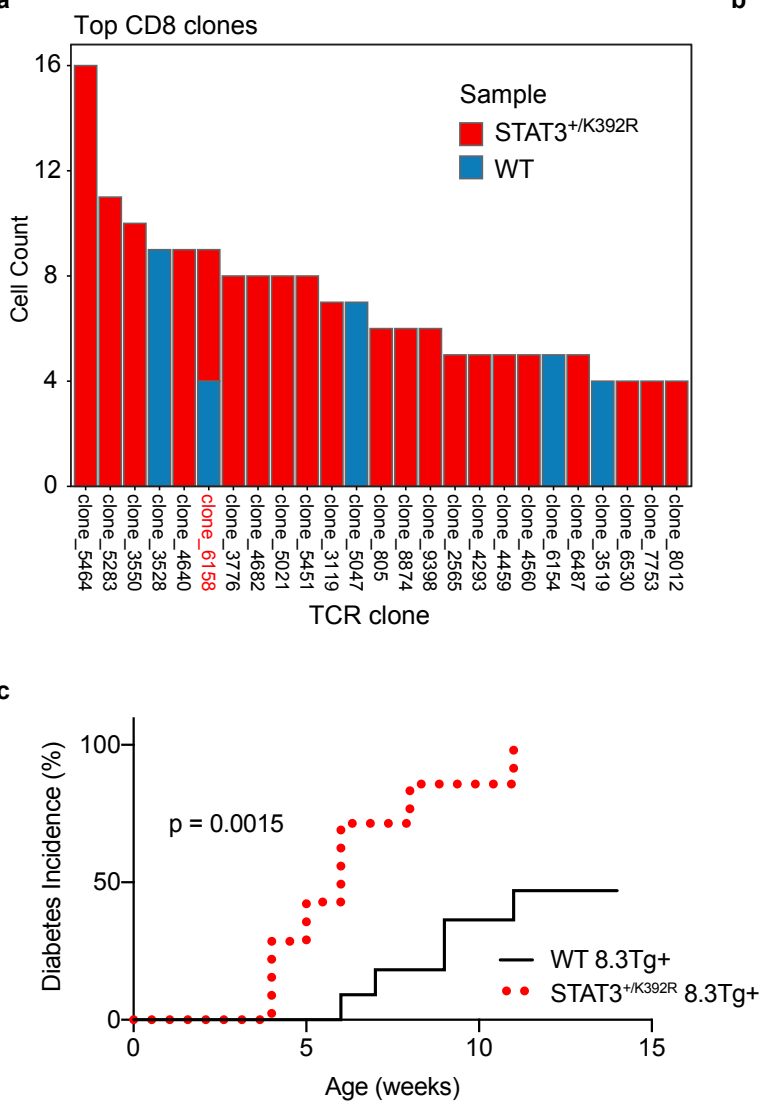

\begin{tabular}{|c|c|c|}
\hline & \multicolumn{2}{|c|}{ CDR3 } \\
\hline Clone & TCR alpha & TCR beta \\
\hline clone_5464 & CALVDGNMGYKLTF & CASSGGQGWNSPLYF \\
\hline clone_5283 & CALSSSSFSKLVF & CASSRVDRDAEQFF \\
\hline clone_3550 & CALALRNSGGSNYKLTF & CASSFGTGGVYEQYF \\
\hline clone_3528 & CAKRNNNNRIFF & CASSFEGQNTLYF \\
\hline clone_4640 & CALSDQGGTGSKLSF & CASSLGWGSYAEQFF \\
\hline clone_6158 & CAMRDSGGSNAKLTF & CASSDSQNTLYF \\
\hline clone_3776 & CALGDRGNTGGLSGKLTF & CASSLDRGARSYEQYF \\
\hline clone_4682 & CALSDRGFASALTF & CASSLTGGRQNTLYF \\
\hline clone_5021 & CALSGPNTGKLTF & CAWSPGTGGADAEQFF \\
\hline clone_5451 & CALVAGTGGYKVVF & CASSLSDRDNSPLYF \\
\hline $\begin{array}{c}\text { TCR-8.3 } \\
\text { (IGRP-reactive) }\end{array}$ & $\frac{\text { TCR alpha }}{\text { CAMRDSGGSNAKLTF }}$ & $\begin{array}{c}\text { TCR beta } \\
\text { CASSDAQNTLYF }\end{array}$ \\
\hline
\end{tabular}

d

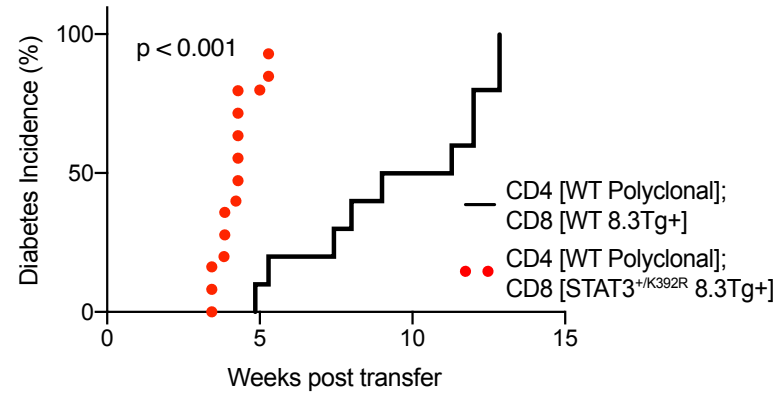


Extended Data Figure 1. Phenotyping of STAT3 ${ }^{+/ K 392 R}$ mouse model. $\mid$. a, Intraperitoneal glucose tolerance testing of young $\left(<8\right.$ weeks of age) WT $(n=11)$ vs STAT3 ${ }^{+/ K 392 R}(n=8)$ mice. $\mathbf{b}$, Immunofluorescence of islet in $\mathrm{STAT}^{+/ K 392 \mathrm{R}} \mathrm{Rag}^{-/-}$showing normal distribution of alpha cells (glucagon + ) and beta cells (insulin + ). Scale bar, $100 \mu \mathrm{m} . \mathbf{c}$, Prevalence of insulin autoantibodies (IAA) in nondiabetic mice 4-8 weeks of age (Females: WT, $\mathrm{n}=20$; STAT3 $^{+/ K 392 R}, n=20$; Males: WT, $n=17$; STAT3 $\left.{ }^{+/ K 392 R}, n=23\right)$. d, H\&E of pancreas from diabetic STAT3 ${ }^{+/ K 392 R}$ mouse at 13 weeks showing severe insulitis. Scale bar, $100 \mu \mathrm{m}$. Yellow arrows indicate dense lymphocytic infiltrate into the islet. e, Lymphoproliferation in non-diabetic female littermates at 6 weeks - spleens and pancreatic lymph nodes as representative examples $\mathbf{f}$, Absolute cell counts in pancreatic lymph node of WT $(n=6)$ vs STAT3 ${ }^{+/ K 392 R}$ $(\mathrm{n}=6)$ non-diabetic mice at 7-8 weeks, $\mathrm{p}=0.006$. $\mathrm{g}$, In vivo Treg suppression assay showing diabetes incidence after adoptive transfer of WT CD4+ BDC2.5+ Teff cells without $(n=6)$ and with Tregs from WT $(n=5)$ or STAT3 ${ }^{+/ K 392 R}$ $(\mathrm{n}=3) \mathrm{BDC} 2.5+$ mice into NOD.Rag $1^{-/-}$mice. Results pooled from 2 independent cohorts. Error bars indicate mean $+/$ SD. ns $=$ non-significant $(\mathrm{P}>0.05), * \mathrm{P} \leq 0.05, * * \mathrm{P} \leq 0.01, * * * \mathrm{P} \leq 0.001$.

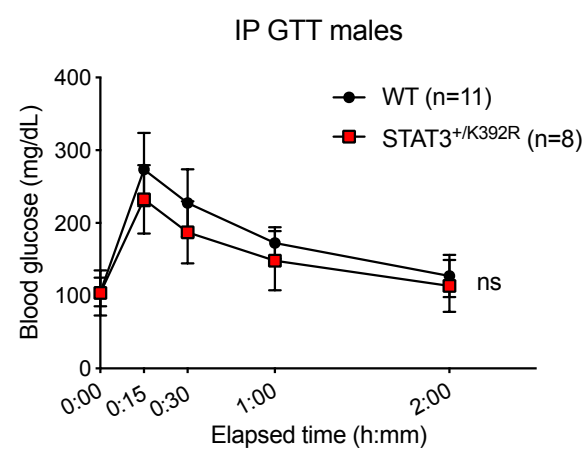

c

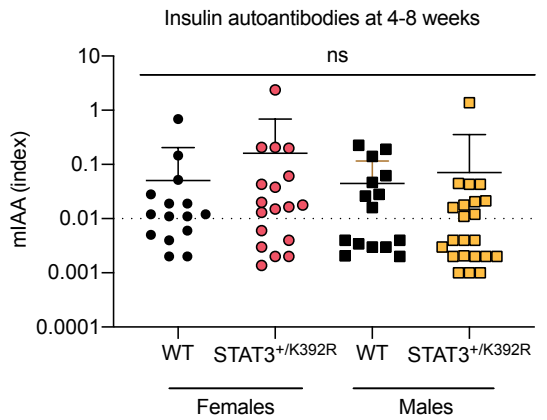

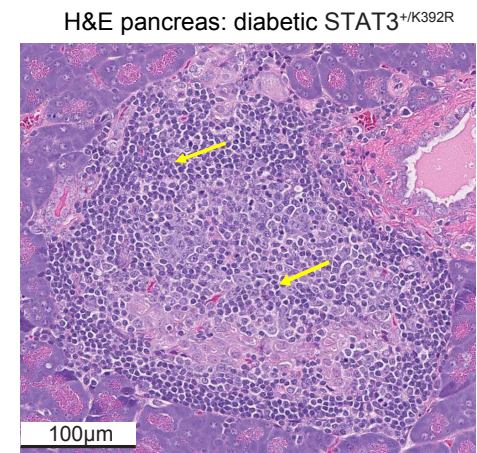

Islet from STAT3 ${ }^{+/ K 392 R}$. RAG $^{-/-}$

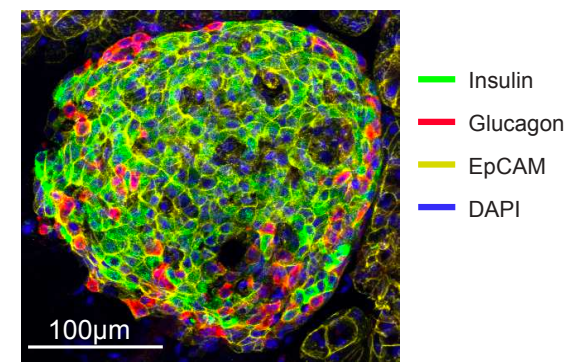

e

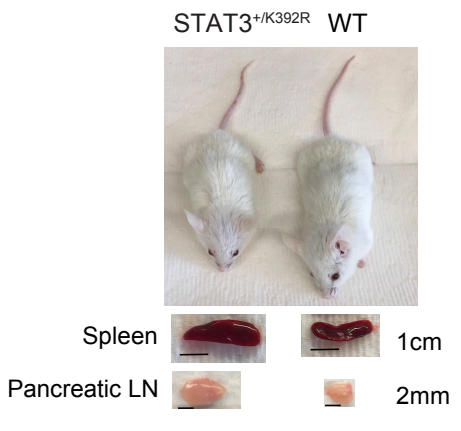

f

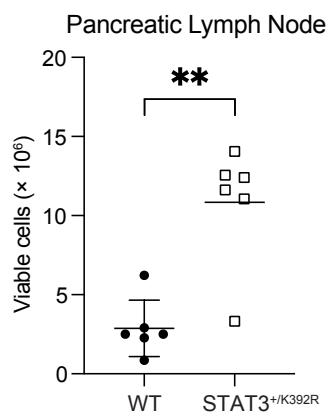

g

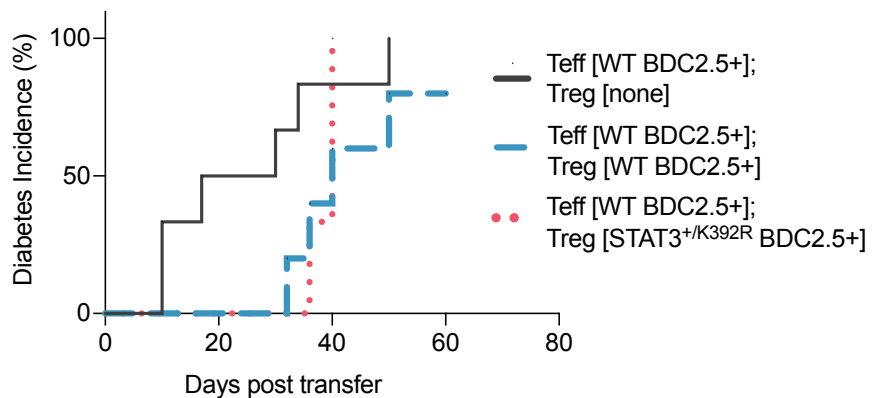


Extended Data Figure 2. scRNA-seq of infiltrating islets additional cluster characterization. | a, UMAP projection of scRNA-seq profiles of cells isolated from CD45+ islet infiltrates of 8-10 week old male non-diabetic mice, WT ( $\mathrm{n}=3$, pooled) vs STAT3 ${ }^{+/ K 392 R}(\mathrm{n}=3$, pooled) $\mathbf{b}$, Summary of marker genes used to identify APC clusters. c, Additional marker gene characterization for the scRNA-seq T cell clusters d, Expression of selected genes visualized for each T cell $\mathbf{e}$, Summary of marker genes used to identify T cell clusters. f, Additional flow cytometry data on GzmA and GzmB protein expression for both genotypes.

a

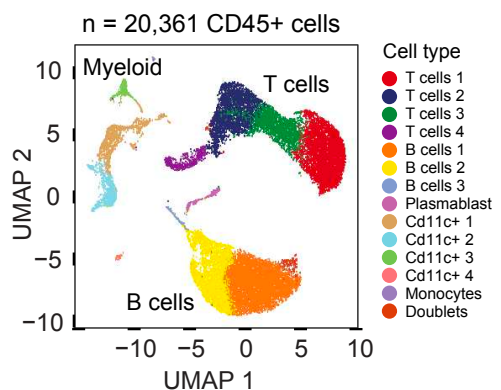

b

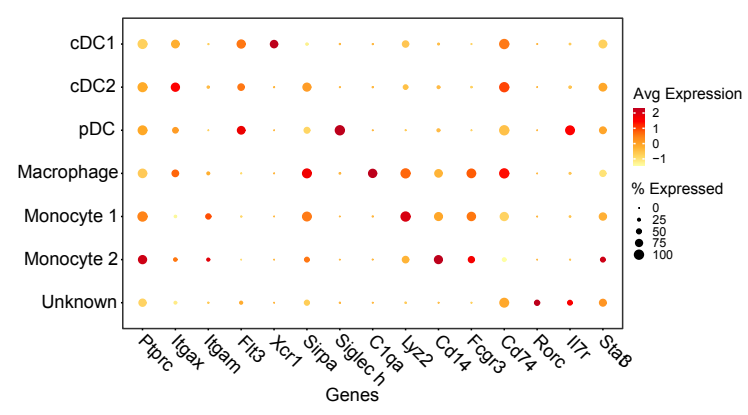

c

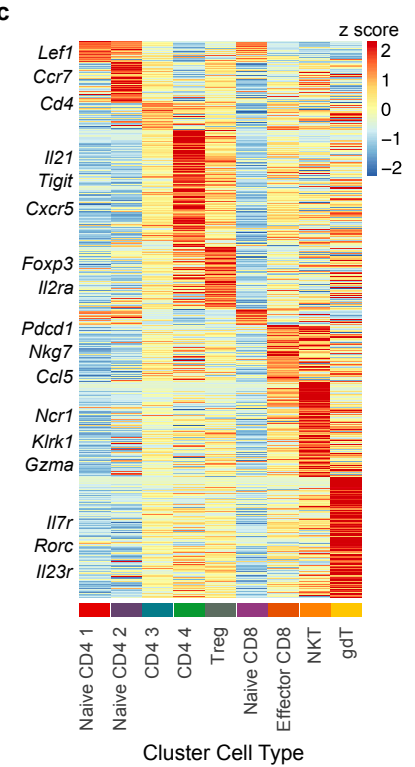

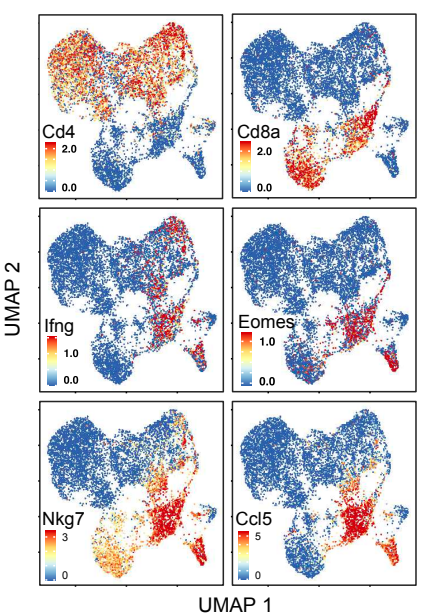

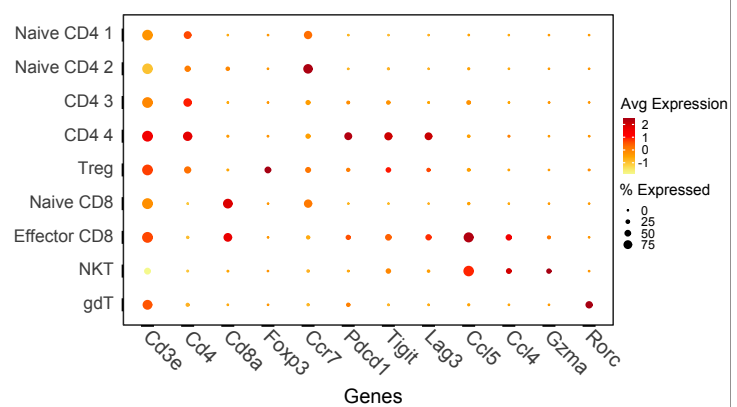

Splenocytes from nondiabetic females at 6 weeks

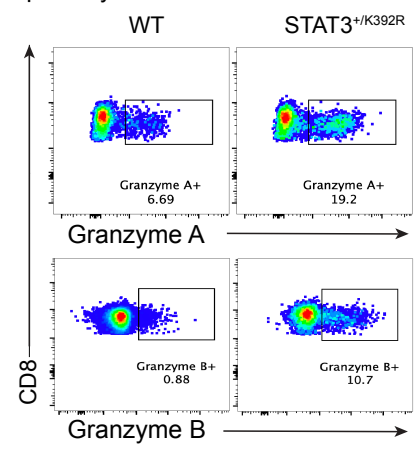

Gated on Live, TCR $\beta+C D 8+$ cells 
Extended Data Figure 3. scATAC-seq of infiltrating islets additional cluster characterization $\mid$ a, aggregated

a

STAT3 $^{+/ K 392 R}$

Fragment Size Distribution

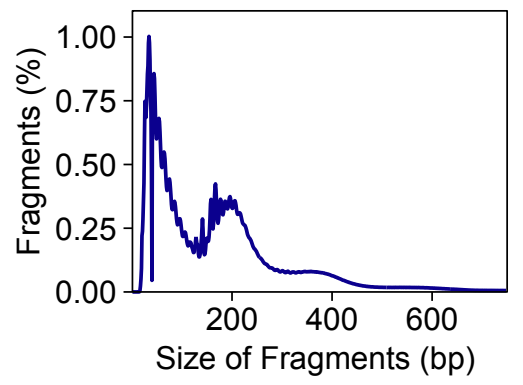

b

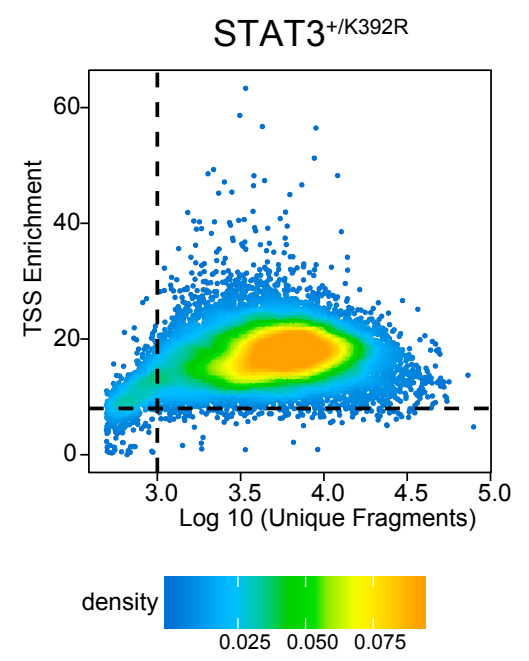

WT

Fragment Size Distribution
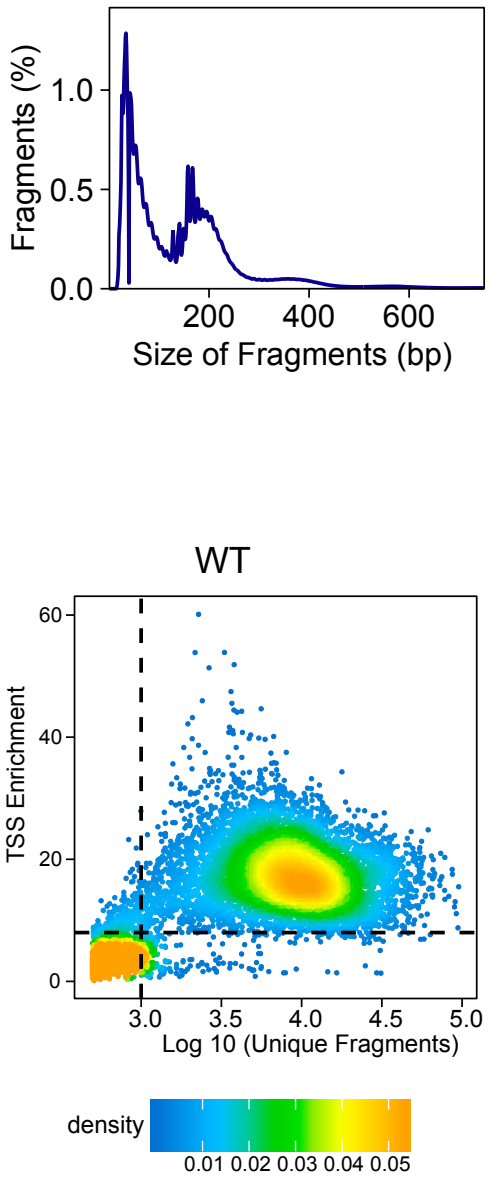

401 
Extended Data Figure 4. Additional characterization of single-cell TCR data from infiltrating islets and functional experiments $\mid$ a, TCR expression in UMAP clusters from subset of CD3+ T cells identified in scRNAseq analysis b, Gini index showing clonal expansion in $\mathrm{STAT}^{+/ K 392 \mathrm{R}}$ broken down by cluster and genotype $\mathbf{c}$, Diabetes incidence after adoptive transfer of naïve $\mathrm{CD} 8+\mathrm{T}$ cells from WT $8.3 \mathrm{Tg}+$ mice with polyclonal $\mathrm{CD} 4+\mathrm{T}$ cells from mice without $(\mathrm{n}=10)$ and with STAT3 ${ }^{+/ K 392 R}(\mathrm{n}=5)$ into NOD.SCID mice d, Diabetes incidence after adoptive transfer of CD4+ BDC2.5+ Teff cells from mice without $(n=6)$ and with STAT3 ${ }^{+/ K 392 R}(n=9)$ into NOD.Rag1 $1^{-/}$mice. Results pooled from 2 independent cohorts.

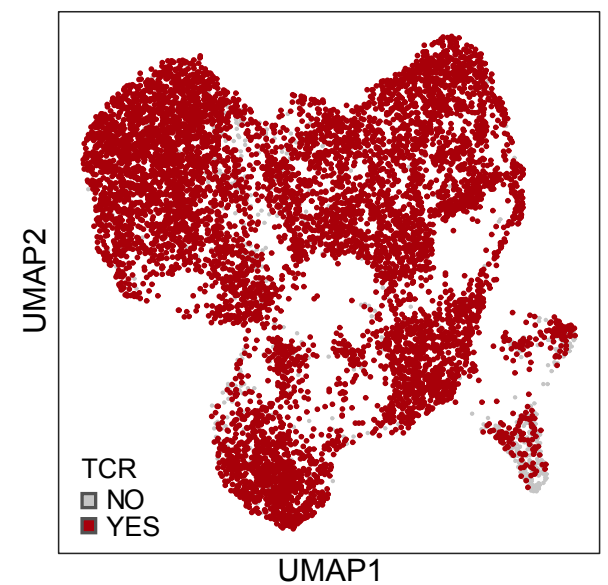

c

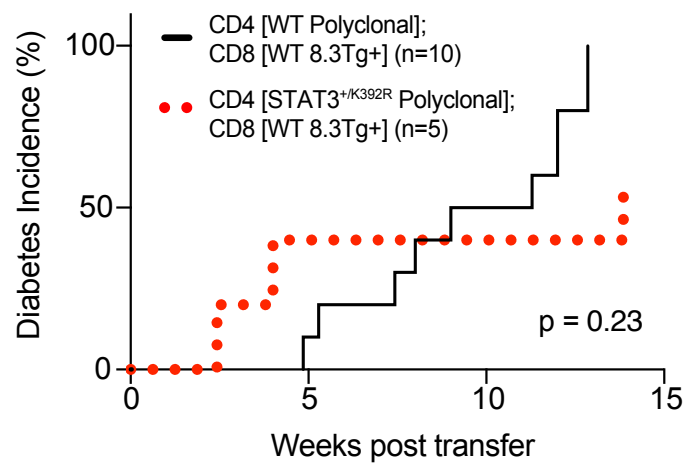

b

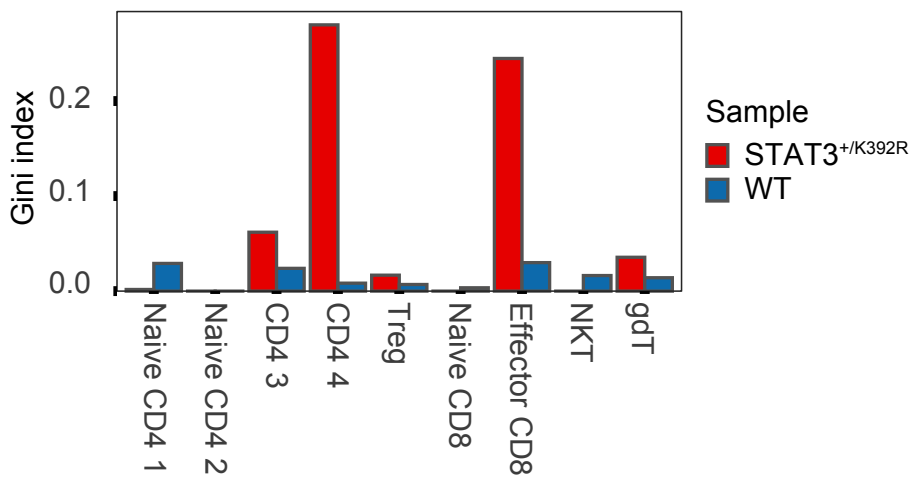

d

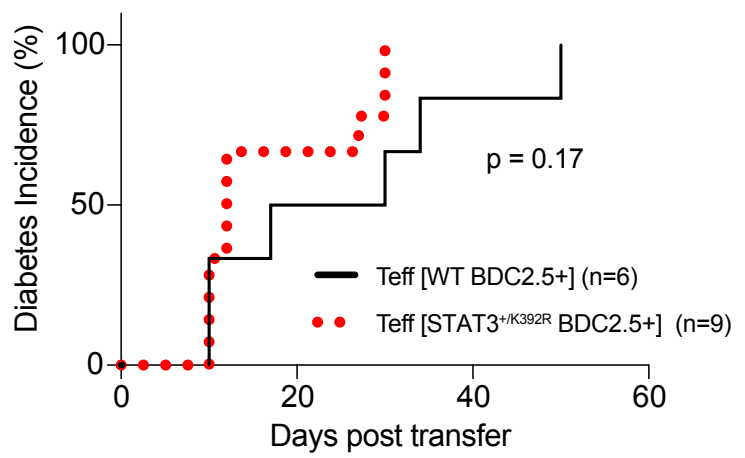




\section{Methods:}

\section{$414 \underline{\text { Mice }}$}

415 The single nucleotide variant 1454 A $>$ G of NM_011486.3 identified in a STAT3 gain-of-function patient was 416 inserted using CRISPR/Cas9 genome editing by The Jackson Laboratory in a custom request ${ }^{46}$. Two founder lines 417 were used for experiments to minimize the risk of off-target effects. Additionally, The Jackson Laboratory provided 418 other mice used in these experiments: NOD (Jax \#001976), NOD.RAG1 ${ }^{-/-}$(Jax \#003729), NOD.SCID (Jax $419 \#$ \#001303), NOD.H2 ${ }^{\mathrm{b} / \mathrm{b}}(\mathrm{Jax} \# 002591)$, NOD.BDC2.5+ (Jax \#004460), NOD.8.3TCR+ (Jax \# 005868) ${ }^{46}$, unless 420 otherwise specified in methods.

421 Mice were maintained in the University of California San Francisco (UCSF) specific pathogen-free animal 422 facility in accordance with the guidelines established by the Institutional Animal Care and Use Committee (IACUC) 423 and Laboratory Animal Resource Center and all experimental procedures were approved by the Laboratory Animal

424 Resource Center at UCSF. Mice aged 4-8 weeks were used for all experiments unless otherwise specified in the text 425 or figure legends. Mice were age-matched in figures displaying a single representative experiment and in pooled 426 data.

\section{Lymphocyte Isolation, Cell Sorting, and Flow cytometry}

429 LN and spleen were isolated by dissection from mice and then mashed through a 70- $\mu \mathrm{m}$ filter. Spleen cells were 430 lysed in ammonium-chloride-potassium lysis buffer to remove red blood cells. Cells isolated from spleen and LNs 431 were counted, and $1-5 \times 10^{6}$ cells were first stained in PBS and Ghost Live/Dead (Tonbo), followed by blocking in 432 2.4G2 before staining with the appropriate antibodies for flow cytometry. For transcription factor staining, cells 433 were fixed overnight in the eBioscience Foxp3/Transcription Factor/Fixation-Concentrate kit (Thermo Fisher 434 Scientific). After fixation, cells were permeabilized and stained with the appropriate antibodies. For ICS, cells were 435 stimulated for $4 \mathrm{~h}$ in Brefeldin A (eBioscience) and eBioscience Cell Stimulation Cocktail $(500 \times)$. Cells were then 436 fixed and permeabilized using the BD cytofix/cytoperm kit before staining with the appropriate antibodies.

437 Islets were purified following standard collagenase protocols as described ${ }^{22}$ and dissociated by incubating with a 438 non-enzymatic solution (Sigma, St, Lois, MO) followed by trituration per the manufacturer's instructions. 
Antibodies used for flow-cytometry were as follows: PE-Cy7-conjugated CD4 (clone RM4-5; Tonbo), PE-Cy7conjugated B220 (clone RA3-6B2; BioLegend), PE-Cy7-conjugated CD62L (clone MEL-14; Tonbo), PE-Cy7-

441 conjugated CD45 (clone 30-F11; Invitrogen), PE-conjugated IL-17A (clone eBio17B7; Invitrogen), PE-conjugated

442 CD62L (clone MEL-14; Tonbo), PE-conjugated CD44 (clone IM7; BioLegend), PE-conjugated CD8 (clone 53-6.7;

443 Tonbo), PE-conjugated ROR $\gamma(\mathrm{Q} 31-378$; BD Biosciences), PE-conjugated IL-13 (clone eBio13A; Invitrogen),

444 FITC-conjugated IFN $\gamma$ (clone xMG1.2; Invitrogen), FITC-conjugated Foxp3 (clone FJK-16s; Invitrogen), FITC445 conjugated CD44 (clone IM7; BioLegend), FITC-conjugated CD8 (clone 53-6.7; Tonbo), APC-conjugated CD25

446 (clone PC61.5; Tonbo), APC-conjugated CD4 (clone GK1.5; BioLegend), APC-conjugated IL-2 (clone JES6-5H4;

447 Invitrogen), APC-conjugated CD62L (clone MEL-14; Tonbo), PerCP-conjugated CD45 (clone 30-F11; Invitrogen), 448 eFluor 450-conjugated TCR $\beta$ (clone H57-597; Tonbo), eFluor 450-conjugated CD8 (clone 53-6.7; Tonbo), PerCP449 Cy5.5-conjugated CD4 (clone GK1.5; BioLegend), Ghost UV 450 (Tonbo), and Ghost 510 (Tonbo).

\section{Cytokine Production}

452 Lymphocyte cells were activated at $10^{6}$ cells $/ \mathrm{ml}$ with $0.5 \mathrm{mM}$ ionomycin, $10 \mathrm{ng} / \mathrm{ml} \mathrm{PMA} \mathrm{and} 3 \mathrm{mg} / \mathrm{ml}$ of Brefeldin 453 A at $37^{\circ} \mathrm{C}$ for 3-4 hour before labeling with LIVE/DEAD fixable dead stain, and staining for CD4, CD8, IL-17 and 454 INF $\gamma$ using Cytofix/Cytoperm.

\section{$\underline{T \text { cell differentiation }}$}

457 T cells were enriched from spleen and LNs using the MagniSort CD4 negative selection kit (Thermo Fisher

458 Scientific). Naive CD4+ T cells were isolated by flow cytometry based on the markers CD4+CD62L+CD44-CD25459 or using the Easysep mouse naive T cell isolation kit. $10^{5}$ naive T cells were cultured for $4 \mathrm{~d}$ (Th1, Th17, iTreg cell) 460 in a 96-well flat-bottom plate coated with $2 \mu \mathrm{g} / \mathrm{ml}$ anti-CD3 (clone 2C11; Tonbo) and $2 \mu \mathrm{g} / \mathrm{ml}$ anti-CD28 (clone 461 37.51; Tonbo) with the relevant cytokines and blocking antibodies: classical Th17 (20 ng/ml IL-6, $2 \mathrm{ng} / \mathrm{ml}$ TGF $\beta, 10$ $462 \mu \mathrm{g} / \mathrm{ml}$ anti-IL4 [clone 11B11; Tonbo], and $10 \mu \mathrm{g} / \mathrm{ml}$ anti-INF $\gamma$ [clone XMG1.2; Tonbo]), pathogenic Th17 (20 $463 \mathrm{ng} / \mathrm{ml}$ IL-6, $20 \mathrm{ng} / \mathrm{ml}$ IL-1 $\beta, 20 \mathrm{ng} / \mathrm{ml} \mathrm{IL-23,} 10 \mu \mathrm{g} / \mathrm{ml}$ anti-IL4, and $10 \mu \mathrm{g} / \mathrm{ml}$ anti-INF $\gamma)$, Th1 (20 ng/ml IL-12, 100 $464 \mathrm{U} / \mathrm{ml}$ IL-2, and $10 \mu \mathrm{g} / \mathrm{ml}$ anti-IL4), iTreg cell (20 ng/ml TGF $\beta$ and $100 \mathrm{U} / \mathrm{ml}$ IL-2), or Th0 (100 U/ml IL-2). Th17 465 cultures were performed in Iscove's medium, Th1/iTreg cell cultures were performed in RPMI. All media were 466 supplemented with 10\% FBS, penicillin/streptomycin, glucose, pyruvate, $\beta$-mercaptoethanol, and Hepes. Cytokines 


\section{Mass Cytometry of Islets}

471 Islets were purified following standard collagenase protocols as described ${ }^{22}$ and dissociated by incubating with a

472 non-enzymatic solution (Sigma, St, Lois, MO) followed by trituration per the manufacturer's instructions. Single

473 cell suspensions were fixed for $10 \mathrm{~min}$ at RT using 1.6\% PFA (Fisher Scientific, Hampton, New Hampshire). Mass-

474 tag cellular barcoding was performed as previously described ${ }^{47}$, followed by sample pooling. Cells were then stained

475 as previously reported ${ }^{48}$. Primary conjugates of mass cytometry antibodies were prepared using the MaxPAR

476 antibody conjugation kit (Fluidigm, South San Francisco, CA) according to the manufacturer's recommended

477 protocol and each antibody clone and lot was titrated to optimal staining concentrations using primary murine

478 samples. Prior to sample acquisition, cells were stained with $125 \mathrm{nM}$ Ir191/193 DNA intercalator (Cell- ID

479 Intercalator-Ir, Fluidigm) for 20min, washed in Di water, filtered through a $35 \mu \mathrm{m}$ nylon mesh and resuspended to

$480 \quad 0.5 \times 10^{6}$ cells $/ \mathrm{ml}$ with $0.1 \%$ EQ four element calibration beads (Fludigm). Data acquisition was done with a CyTOF

4812 mass cytometer (Fluidigm) at an event rate of 300-500 cells/s. After data acquisition, .fcs files were concatenated,

482 normalized using mass bead signal ${ }^{49}$ and debarcoded using a single-cell debarcoding algorithm ${ }^{47}$. Manual gating and 483 viSNE analysis of .fcs files was performed using Cytobank ${ }^{50}$.

\section{Bone Marrow Chimeras}

486 Bone marrow was removed from 4-8 week nondiabetic, CD45.2 congenically labelled donor mice, filtered through a

487 70-mm filter, centrifuged, and resuspended in sterile PBS to a concentration of $10^{7}$ live cells per $200 \mu 1$. CD45.1

488 congenically labelled female NOD recipient mice were lethally irradiated (1200 rad) 8-16 h before bone marrow 489 transplant. A single-cell suspension of CD45.2 bone marrow in sterile PBS ( $10^{7}$ live cells per recipient mouse) was

490 transplanted to each recipient mouse by IV tail injection. Reconstitution was confirmed by flow cytometry of the

491 peripheral blood at 4-8 weeks post transplantation. Nonfasting blood glucose levels in recipient mice were

492 monitored weekly by using an Accu-Check glucometer (Roche Diagnostic Corp). Diabetes onset was considered to

493 have occurred when nonfasting blood glucose concentration exceeded $250 \mathrm{mg} / \mathrm{dl}$ for 2 consecutive days. 
496 NOD.BDC2.5+ and NOD.BDC2.5+STAT3 ${ }^{+/ K 392 \mathrm{R}}$ lymphocyte single cell suspensions were made from axillary,

497 inguinal, mesenteric and para-aortic LNs, as discussed above. CD4+CD25+CD62L+ Tregs and CD4+CD25-

498 CD62L + Teffs were isolated by cell sorting using a FACS Aria sorter, prior to being incubated in ex-vivo cultures at

$49937^{\circ} \mathrm{C}$ for ten days. $25-50 \times 10^{3}$ purified cells were plated on 96-well U-bottom plate, stimulated with mouse anti-

$500 \mathrm{CD} 3 / \mathrm{CD} 28$ dynabeads (3:1 bead to cell ratio; Thermo Fisher Scientific, Inc.) and supplemented with complete

501 medium consisting of DMEM containing 10\% heat-inactivated fetal bovine serum (Biosource International), $5 \mu \mathrm{M}$

502 HEPES, nonessential amino acids, $0.5 \mathrm{mM}$ sodium pyruvate, $1 \mathrm{mM}$ glutaMax I (all from Invitrogen), penicillin-

503 streptomycin, recombinant human IL-2 (2000IU/mL for Treg and 200IU/mL for Teff cultures; Proleukin) and $55 \mu \mathrm{M}$

504 2-ME (Sigma-Aldrich). On day ten of ex-vivo culture, $5 \times 10^{6}$ expanded NOD.BDC2.5+ or

505 NOD.BDC2.5+STAT3 ${ }^{+/ K 392 R}$ Tregs along with $5 \times 10^{6}$ NOD.BDC2.5+ Teffs were harvested, washed twice, rested in

506 complete media without any IL-2 supplementation for 6 hours at $37^{\circ} \mathrm{C}$, and resuspended in $100 \mu \mathrm{L}$ of PBS before the

507 individual cell suspensions were injected intravenously via the retrorbital vein into anesthetized NOD.Rag2 $2^{-/ 2}$ mice.

508 Successful intravenous infusion was confirmed by direct visualization and palpation of the periorbital area.

509 Nonfasting blood glucose levels in recipient mice were monitored daily by using an Accu-Check glucometer (Roche

510 Diagnostic Corp). Diabetes onset was considered to have occurred when nonfasting blood glucose concentration

511 exceeded $250 \mathrm{mg} / \mathrm{dl}$ for 3 consecutive days.

\section{2}

\section{$513 \quad$ Adoptive Transfer of Diabetes using 8.3Tg+ CD8+ T cells}

514 NOD, NOD.8.3Tg+ and NOD.8.3Tg+STAT3 ${ }^{+/ K 392 R}$ lymphocyte single cell suspensions were made from

515 nonpancreatic lymph nodes and spleen from nondiabetic donors, as discussed above. NOD cells were CD4-enriched

516 using MACS EasySep CD4-negative selection kits (StemCell), and 8.3Tg+ cells were naïve CD8-enriched using

517 MACS EasySep naïve CD8-negative selection kits (StemCell). Aliquots at each step were analyzed to confirm

518 purity. Purified cells were pooled in a 1:2 ratio of naïve CD8+8.3Tg+ T cells to polyclonal CD4+ T cells, and

519 retrorbitally injected at $1.5 \times 10^{6}$ cells per recipient NOD.SCID mouse. Nonfasting blood glucose levels in recipient

520 mice were monitored three times per week by using an Accu-Check glucometer (Roche Diagnostic Corp) from days

521 7-30 post-transfer. Diabetes onset was considered to have occurred when nonfasting blood glucose concentration

522 exceeded $250 \mathrm{mg} / \mathrm{dl}$ for 2 consecutive days. 
525 NOD.BDC2.5+.Foxp $3^{\text {RFP }}$ nondiabetic mice donated from the Tang Lab at UCSF were used to generate lymphocyte

526 single cell suspensions from nonpancreatic lymph nodes and spleen, as discussed above.

527 CD4+CD62L+Vß4+Foxp3- cells were isolated by cell sorting using a FACS Aria sorter, labeled using the

528 Invitrogen CellTrace Violet Proliferation Kit, and $1.0 \times 10^{6} \mathrm{CTV}$-labeled cells were injected i.v. into WT or

$529 \mathrm{STAT}^{+/ K 392 \mathrm{R}}$ nondiabetic recipients. 3.5 days post-adoptive transfer, pancreatic and inguinal lymph nodes were

530 removed and analyzed for the presence of CTV-labeled cells to assess cell proliferation.

\section{Single cell RNA-seq library preparation and sequencing}

533 Islets were purified following standard collagenase protocols as described ${ }^{22}$ and dissociated by incubating with a non-enzymatic solution (Sigma, St, Lois, MO) followed by trituration per the manufacturer's instructions. After live

535 CD45+ cells were isolated by cell sorting using a FACS Aria sorter, cells were spun down and resuspended in PBS.

536 Prior to loading on the 10x Chromium instrument, cells were counted using a haemocytometer and the concentration 537 of cells adjusted to $\sim 1 \times 10^{3}$ cells $\mu \mathrm{L}^{-1}$. A viability of at least $90 \%$ for all samples were confirmed by trypan blue 538 staining. Samples were handled on ice where possible. Cells were then processed by the UCSF Immunology Core 539 facility using the Chromium Single Cell 5' Library and the Gel Bead Kit following the manufacturer's user guide 540 (10x Genomics, Pleasanton, CA, CG000086_SingleCellVDJReagentKitsUserGuide_RevB). Single-cell libraries 541 were sequenced on a Novaseq S4 Flowcell PE 2x150.

\section{Single cell RNA-seq analysis}

544 Reads were processed and aligned to the mm10 reference genome assembly using the 10X cellranger count pipeline.

545 Doublets were assessed using the R implementation of scrublet ${ }^{51}$, 'rscrublet'. Seurat objects for each sample were 546 created and merged. Barcodes with a doublet score greater than 0.15 , mitochondrial reads greater than $5 \%$, or less 547 than 200 features were removed.

549 Pre-processing, clustering, and dimensionality reduction were performed using Seurat ${ }^{52}$. Clusters were identified 550 using marker genes including $C d 3 e, C d 19$, and Itgax. T cell clusters 1, 2, and 3 were selected for re-clustering and 
551 were subsequently re-processed using the same procedure. A small number of additional contaminants (doublets and

552 non-T cells) were identified and removed. Differential genes were determined with Seurat's `FindAllMarkers` and

553 'FindMarkers' functions and represented as heatmaps (plotted with 'pheatmap') or volcano plots (plotted with

554 'ggplot2'). For clarity, ribosomal genes, mitochondrial genes, genes starting with "Gm”, and genes ending with

555 "Rik", are not shown in the volcano plots.

$557 \quad$ Single cell TCR analysis

558 TCR sequences were aligned to the mm10 reference genome using the 10X cellranger vdj pipeline. High confidence

559 clonotypes for each sample were merged to obtain a single clone id by matching identical CDR3 amino acid

560 sequences between the samples. These merged clones were added to the Seurat object metadata for downstream

561 analysis

562

\section{Single cell ATAC-seq library preparation and sequencing}

564 Cells were processed using the 10X single cell ATAC reagents and protocols as described previously ${ }^{53}$. Briefly, bulk

565 cells were transposed, single cells were loaded into droplets for barcoding using the 10X chromium platform, and

566 then DNA was amplified and prepared for sequencing. Libraries were sequenced at the Stanford Functional

567 Genomics Facility.

568

\section{$569 \quad$ Single cell ATAC analysis}

570 Reads were processed and aligned to the mm10 reference genome assembly using the 10X cellranger atac-count

571 pipeline. Fragments files were loaded into ArchR for all downstream analysis ${ }^{54}$. ArchR default settings were used

572 for doublet calling, quality filtering (based on transcription start site enrichment and number of fragments per cell)

573 and computing the cell by tile matrix.

574

575 The tile matrix was used for dimensionality reduction and clustering of the cells. ArchR gene scores were used to

576 determine cluster identities and T cells were selected for re-clustering, analogous to the scRNA workflow. ArchR

577 was then used to create a cell by peak matrix and cell by motif deviation matrix. Markers for each matrix were

578 determined using the 'getMarkerFeatures` utility and then displayed via heatmap or volcano plot. Accessibility for 
selected genomic regions were visualized using the ArchR browser where each track for a particular region is shown on the same fixed scale and normalized by reads in transcription start sites genome-wide.

582 Histology

583 Pancreata were removed and fixed in 10\% neutral-buffered formalin (Sigma-Aldrich). Paraffin-embedded step-

584 sections were stained with hematoxylin and eosin (H\&E) and insulitis was scored as described previously ${ }^{55}$.

\section{$\underline{\text { Immunofluorescence }}$}

587 For immunofluorescence, panncreata were fixed in $2 \%$ paraformaldehyde (Pierce) in PBS for $2 \mathrm{hrs}$ at $4{ }^{\circ} \mathrm{C}$ followed 588 by overnight incubation in 30\% (w/v) sucrose (Sigma Aldrich) in PBS. Tissues were embedded in Optimal Cutting

589 Temperature Compound (Tissue-Tek) and stored at $-80^{\circ} \mathrm{C}$ before sectioning $(50-200 \mu \mathrm{m})$ on a cryostat (Leica).

590 Thin sections were dried on Superfrost Plus (Fisher Scientific) slides and semi-thick $(200 \mu \mathrm{m})$ sections were moved 591 directly to $0.3 \%$ Triton X-100 (Sigma-Aldrich), 0.2\% BSA (Sigma-Aldrich), 0.1\% sodium azide (Sigma-Aldrich) in

592 PBS (Immunomix). Slides were stained in a humidified chamber and semi-thick sections were stained in 24-well 593 plates with one section per well. Slides were briefly rehydrated in PBS before permeabilization in Immunomix for $5941 \mathrm{hr}$ at RT followed blocking with BlockAid (ThermoFisher), primary antibody staining at RT, and, when necessary, 595 secondary antibody staining at RT for $1 \mathrm{hr}$. Semi-thick sections were permeabilized in Immunomix with shaking at 596 RT overnight followed by blocking with BlockAid at RT for 2hr, primary antibody staining at RT for $2 \mathrm{hr}$, and, when 597 necessary, secondary antibody staining at RT for $2 \mathrm{hr}$. Semi-thick sections were then moved to Superfrost Plus slides 598 and all sections were mounted with ProLong Diamond Antifade Mountant (ThermoFisher). Images were acquired 599 on a Leica SP5 (Leica) laser scanning confocal microscope.

\section{$601 \quad$ Statistical analysis}

602 All experiments were performed using randomly assigned mice without investigator blinding. No data were

603 excluded. Statistical significance between two groups was calculated using an unpaired, parametric, 2-tailed

604 Student's $\mathrm{t}$ test. Experimental groups included a minimum of three biological replicates. Intragroup variation was 605 not assessed. All statistical analysis was performed using Prism 7 (GraphPad Software). Figures display 

methods were used to predetermine sample size.

\section{References:}

610 1. Gepts, W. \& De Mey, J. Islet cell survival determined by morphology. An immunocytochemical study of 611 the islets of Langerhans in juvenile diabetes mellitus. Diabetes 27 Suppl 1, 251-261 (1978).

612

613

614

2. Gepts, W. Pathologic Anatomy of the Pancreas in Juvenile Diabetes Mellitus. Diabetes 14, 619-633 (1965).

616

617

3. Erlich, H. et al. HLA DR-DQ Haplotypes and Genotypes and Type 1 Diabetes Risk. Diabetes 57 (2008).

4. Fung, E.Y.M.G. et al. Analysis of 17 autoimmune disease-associated variants in type 1 diabetes identifies 6q23/TNFAIP3 as a susceptibility locus. Genes And Immunity 10, 188 (2008).

5. Ferreira, R.C. et al. Functional IL6R 358Ala allele impairs classical IL-6 receptor signaling and influences risk of diverse inflammatory diseases. PLoS Genet 9, e1003444 (2013).

6. Wang, J. et al. Common variants on cytotoxic T lymphocyte antigen-4 polymorphisms contributes to type 1 diabetes susceptibility: evidence based on 58 studies. PloS one 9, e85982 (2014).

7. Sharma, A. et al. Identification of non-HLA genes associated with development of islet autoimmunity and type 1 diabetes in the prospective TEDDY cohort. Journal of autoimmunity 89, 90-100 (2018).

8. Warshauer, J.T., Bluestone, J.A. \& Anderson, M.S. New Frontiers in the Treatment of Type 1 Diabetes.

631

632

633

634

635

636

637

638

639

640

641

642

643

644

9. Flanagan, S.E. et al. Activating germline mutations in STAT3 cause early-onset multi-organ autoimmune disease. Nature genetics 46, 812-814 (2014).

10. Velayos, T. et al. An Activating Mutation in STAT3 Results in Neonatal Diabetes Through Reduced Insulin Synthesis. Diabetes 66, 1022-1029 (2017).

11. O'Shea, John J. \& Plenge, R. JAK and STAT Signaling Molecules in Immunoregulation and ImmuneMediated Disease. Immunity 36, 542-550 (2012).

12. Saarimäki-Vire, J. et al. An Activating STAT3 Mutation Causes Neonatal Diabetes through Premature Induction of Pancreatic Differentiation. Cell Reports 19, 281-294 (2017).

13. Gorogawa, S. et al. Insulin secretory defects and impaired islet architecture in pancreatic beta-cell-specific STAT3 knockout mice. Biochem Biophys Res Commun 319, 1159-1170 (2004).

14. Fabbri, M., Frixou, M., Degano, M. \& Fousteri, G. Type 1 Diabetes in STAT Protein Family Mutations: Regulating the Th17/Treg Equilibrium and Beyond. Diabetes 68, 258-265 (2019).

15. Milner, J.D. et al. Early-onset lymphoproliferation and autoimmunity caused by germline STAT3 gain-offunction mutations. Blood 125, 591-599 (2015).

16. Marwaha, A.K. et al. Cutting Edge: Increased IL-17-Secreting T Cells in Children with New-Onset Type 1 Diabetes. The Journal of Immunology 185, 3814 (2010). 
17. Viisanen, T. et al. Circulating CXCR5\&lt;sup\&gt;+\&lt;/sup\&gt;PD1\&lt;sup\&gt;+\&lt;/sup\&gt;ICOS\&lt;sup\&gt;+\&lt;/sup\&gt; Follicular T Helper Cells Are Increased Close to the Diagnosis of Type 1 Diabetes in Children With Multiple Autoantibodies. Diabetes 66, 437 (2017).

18. Hundhausen, C. et al. Enhanced T cell responses to IL-6 in type 1 diabetes are associated with early clinical disease and increased IL-6 receptor expression. Science translational medicine 8, 356ra119 (2016).

19. Wienke, J. et al. A novel human STAT3 mutation presents with autoimmunity involving Th17 hyperactivation. Oncotarget 6 (2015).

20. Durant, L. et al. Diverse targets of the transcription factor STAT3 contribute to T cell pathogenicity and homeostasis. Immunity 32, 605-615 (2010).

21. Stadinski, B.D. et al. Chromogranin A is an autoantigen in type 1 diabetes. Nature immunology 11, 225231 (2010).

22. Tang, Q. et al. In vitro-expanded antigen-specific regulatory T cells suppress autoimmune diabetes. The Journal of experimental medicine 199, 1455-1465 (2004).

23. Tarbell, K.V., Yamazaki, S., Olson, K., Toy, P. \& Steinman, R.M. CD25+ CD4+ T cells, expanded with dendritic cells presenting a single autoantigenic peptide, suppress autoimmune diabetes. The Journal of experimental medicine 199, 1467-1477 (2004).

24. Hudson, W.H. et al. Proliferating Transitory T Cells with an Effector-like Transcriptional Signature Emerge from PD-1+ Stem-like CD8+ T Cells during Chronic Infection. Immunity 51, 1043-1058.e1044 (2019).

25. Schep, A.N., Wu, B., Buenrostro, J.D. \& Greenleaf, W.J. chromVAR: inferring transcription-factorassociated accessibility from single-cell epigenomic data. Nature Methods 14, 975-978 (2017).

26. Kaech, S.M. \& Cui, W. Transcriptional control of effector and memory CD8+ T cell differentiation. Nature Reviews Immunology 12, 749-761 (2012).

27. Milner, J.J. et al. Runx3 programs CD8+ T cell residency in non-lymphoid tissues and tumours. Nature 552, 253-257 (2017).

28. Weinreich, M.A. et al. KLF2 transcription-factor deficiency in T cells results in unrestrained cytokine production and upregulation of bystander chemokine receptors. Immunity 31, 122-130 (2009).

29. Verdaguer, J. et al. Spontaneous autoimmune diabetes in monoclonal T cell nonobese diabetic mice. The Journal of experimental medicine 186, 1663-1676 (1997).

30. Jun, H.S., Santamaria, P., Lim, H.W., Zhang, M.L. \& Yoon, J.W. Absolute requirement of macrophages for the development and activation of beta-cell cytotoxic CD8+ T-cells in T-cell receptor transgenic NOD mice. Diabetes 48, 34-42 (1999).

31. De Leenheer, E. \& Wong, F.S. Adoptive Transfer of Autoimmune Diabetes Using Immunodeficient Nonobese Diabetic (NOD) Mice. Methods in molecular biology (Clifton, N.J.) 1433, 135-140 (2016).

32. Christianson, S.W., Shultz, L.D. \& Leiter, E.H. Adoptive transfer of diabetes into immunodeficient NOD$\mathrm{scid} / \mathrm{scid}$ mice. Relative contributions of CD4+ and CD8+ T-cells from diabetic versus prediabetic NOD.NON-Thy-1a donors. Diabetes 42, 44-55 (1993).

33. Cui, W., Liu, Y., Weinstein, J.S., Craft, J. \& Kaech, S.M. An interleukin-21-interleukin-10-STAT3 pathway is critical for functional maturation of memory CD8+ T cells. Immunity 35, 792-805 (2011). 
34. Ciucci, T., Vacchio, M.S. \& Bosselut, R. A STAT3-dependent transcriptional circuitry inhibits cytotoxic gene expression in T cells. Proceedings of the National Academy of Sciences of the United States of America 114, 13236-13241 (2017).

35. Beltra, J.C. et al. Developmental Relationships of Four Exhausted CD8(+) T Cell Subsets Reveals Underlying Transcriptional and Epigenetic Landscape Control Mechanisms. Immunity 52, 825-841.e828 (2020).

36. Siddiqui, I. et al. Intratumoral Tcf1+PD-1+CD8+ T Cells with Stem-like Properties Promote Tumor Control in Response to Vaccination and Checkpoint Blockade Immunotherapy. Immunity 50, 195-211.e110 (2019).

37. Im, S.J. et al. Defining CD8+ T cells that provide the proliferative burst after PD-1 therapy. Nature 537, 417-421 (2016).

38. Wu, T. et al. The TCF1-Bcl6 axis counteracts type I interferon to repress exhaustion and maintain T cell stemness. Science Immunology 1, eaai8593 (2016).

39. McKinney, E.F., Lee, J.C., Jayne, D.R., Lyons, P.A. \& Smith, K.G. T-cell exhaustion, co-stimulation and clinical outcome in autoimmunity and infection. Nature 523, 612-616 (2015).

40. Keir , M.E. et al. Tissue expression of PD-L1 mediates peripheral T cell tolerance. Journal of Experimental Medicine 203, 883-895 (2006).

41. Ansari, M.J. et al. The programmed death-1 (PD-1) pathway regulates autoimmune diabetes in nonobese diabetic (NOD) mice. The Journal of experimental medicine 198, 63-69 (2003).

42. Wiedeman, A.E. et al. Autoreactive CD8+ T cell exhaustion distinguishes subjects with slow type 1 diabetes progression. The Journal of clinical investigation 130, 480-490 (2020).

43. Long, S.A. et al. Partial exhaustion of CD8 T cells and clinical response to teplizumab in new-onset type 1 diabetes. Sci Immunol 1 (2016).

44. Herold, K.C. et al. An Anti-CD3 Antibody, Teplizumab, in Relatives at Risk for Type 1 Diabetes. New England Journal of Medicine (2019).

45. June, C.H., Warshauer, J.T. \& Bluestone, J.A. Is autoimmunity the Achilles' heel of cancer immunotherapy? Nature medicine 23, 540-547 (2017).

46. Madisen, L. et al. A robust and high-throughput Cre reporting and characterization system for the whole mouse brain. Nat Neurosci 13, 133-140 (2010).

47. Zunder, E.R., Lujan, E., Goltsev, Y., Wernig, M. \& Nolan, G.P. A continuous molecular roadmap to iPSC reprogramming through progression analysis of single-cell mass cytometry. Cell stem cell 16, 323-337 (2015).

48. Levine, L. et al. Single-cell metabolic analysis by mass cytometry reveals distinct transitional states of CD8 T cell differentiation, 2020.

49. Finck, R. et al. Normalization of mass cytometry data with bead standards. Cytometry A 83, 483-494 (2013).

50. Kotecha, N., Krutzik, P.O. \& Irish, J.M. Web-based analysis and publication of flow cytometry experiments. Curr Protoc Cytom Chapter 10, Unit10.17 (2010). 
51. Wolock, S.L., Lopez, R. \& Klein, A.M. Scrublet: Computational Identification of Cell Doublets in SingleCell Transcriptomic Data. Cell Syst 8, 281-291.e289 (2019).

770

771

772

773

774

775

776

777

778

779

780

52. Stuart, T. et al. Comprehensive Integration of Single-Cell Data. Cell 177, 1888-1902.e1821 (2019).

53. Satpathy, A.T. et al. Massively parallel single-cell chromatin landscapes of human immune cell development and intratumoral T cell exhaustion. Nature biotechnology 37, 925-936 (2019).

54. Granja, J.M. et al. ArchR: An integrative and scalable software package for single-cell chromatin accessibility analysis. bioRxiv, 2020.2004.2028.066498 (2020).

55. Katz, J.D., Wang, B., Haskins, K., Benoist, C. \& Mathis, D. Following a diabetogenic T cell from genesis through pathogenesis. Cell 74, 1089-1100 (1993).

781

782 


\section{Acknowledgements:}

784 We thank M. Waterfield, C. Miller, and members of the Anderson laboratories for helpful discussions; K. Wu for

785 experimental support. We thank V. Nguyen for assistance with islet isolations and flow cytometry experiments. We

786 thank S. Tamaki, K. Hiam, M. Spitzer for assistance with mass cytometry experiments. This work was supported by

787 NIH Medical Scientist Training Program grant 2T32DK007418-36A1 to UCSF (School of Medicine); Endocrine

788 Fellows Foundation (JTW); NIH Diabetes Research Center grant P30 DK063720 (A.C.F., M.S.A., Single Cell

789 Analysis Center); NIH Shared Instrument Grant 1S10OD021822-01 (Single Cell Analysis Center). M.S.A. was

790 supported in part by the Larry L. Hillblom Foundation and the Juvenile Diabetes Research Foundation Center of

791 Excellence in Northern California. A.T.S. was supported by a Bridge Scholar Award from the Parker Institute for

792 Cancer Immunotherapy, a Technology Impact Award from the Cancer Research Institute, a Career Award for

793 Medical Scientists from the Burroughs Wellcome Fund, and NIH grant K08CA230188.

795 Author Contributions:

796 J.T.W. conceived the study, designed and performed experiments, analyzed data and wrote the manuscript. J.A.B.

797 designed and performed biostatistical analysis of RNA, TCR, and ATAC sequencing data. A.C conceived and

798 designed experiments with J.T.W, performed flow cytometry experiments and analyzed data. Q.S. performed

799 genomic experiments. N.S and Y.P. designed, performed and analyzed data for the in vivo Treg suppression assay

800 with Q.T. supervision and guidance. J.W and A.G. designed, performed and analyzed data for CD8 adoptive

801 transfer experiments with J.G. supervision and guidance. J.P. designed and performed flow cytometry experiments.

802 A.T.S. supervised genomic experiments and analysis. M.S.A directed the study and wrote the manuscript with

803 J.T.W.

804 Competing financial interests:

805 A.T.S. is a scientific founder of Immunai and receives research funding from Arsenal Biosciences.

806 Materials \& Correspondence:

Mark S. Anderson (mark.anderson@ucsf.edu) 


\section{Figures}

a

STAT3

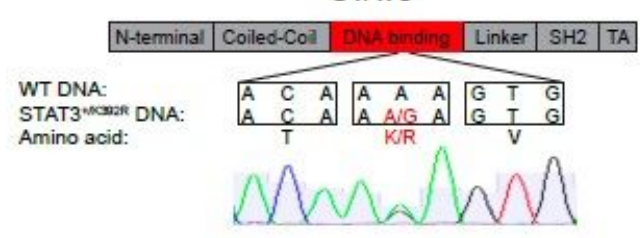

b

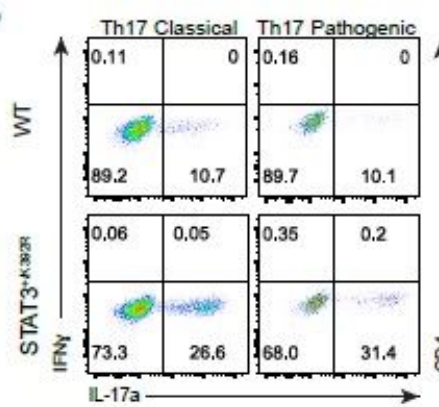

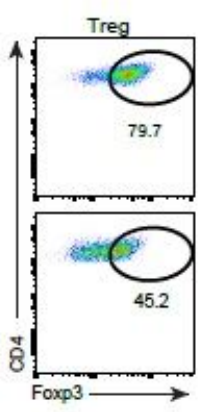

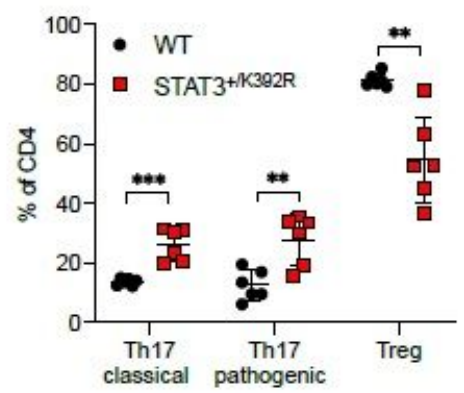

C Diabetes Incidence WT vs STAT3+13392R
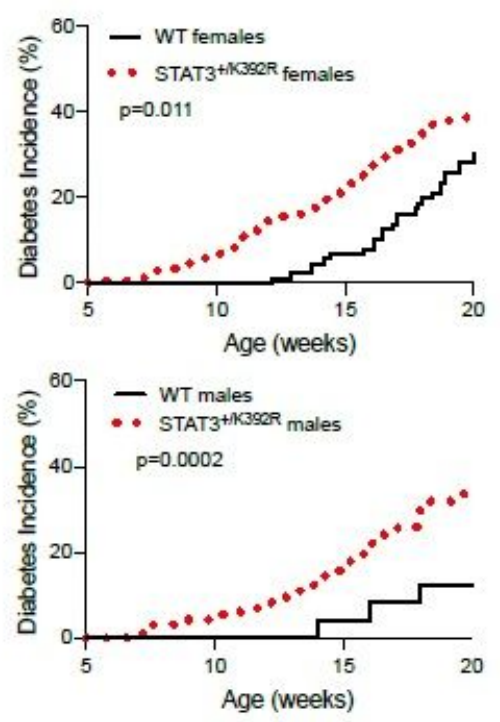

d

e

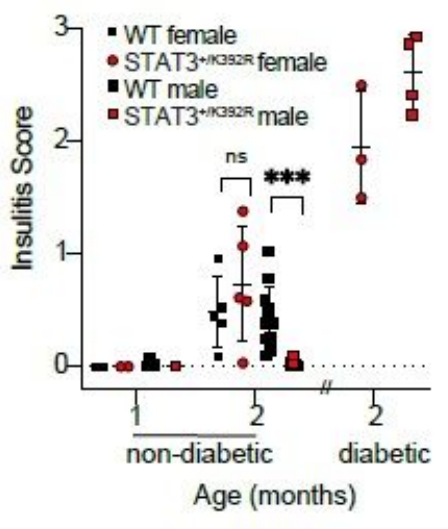

Mass cytometry of islets

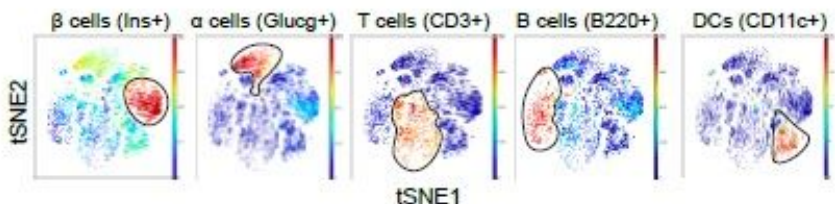

tSNE1

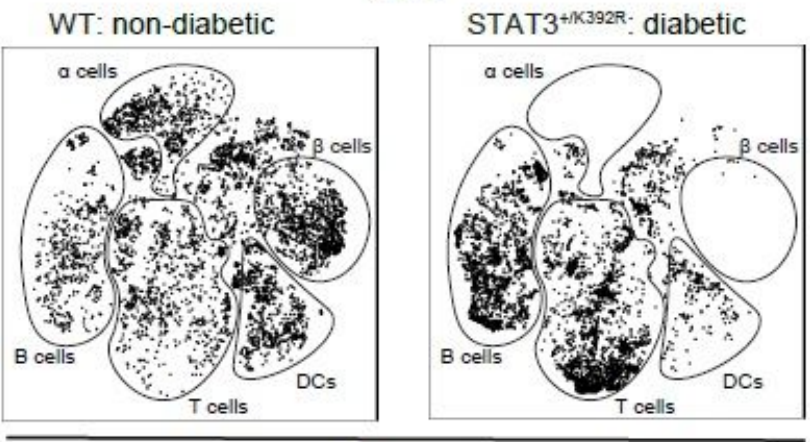

8 weeks

\section{Figure 1}

STAT3+/K392R mutant mice recapitulate the human type 1 diabetes phenotype I a, STAT3K392R mutation located in the DNA binding domain was inserted into WT (i.e., NOD) mice using CRISPR/Cas9 and confirmed by Sanger sequencing b, In vitro CD4 differentiation of naïve CD4+ T cells into Th17 and Treg subsets. c, Diabetes incidence curves (Females: WT, n=93; STAT3+/K392R, n=125; Males: WT, $n=19$; STAT3+/K392R, $n=68) d$, Time course of insulitis in WT and STAT3+/K392R mice with and without diabetes ( $n=3-11$ mice per gender per time point) e, viSNE plots of islets using mass cytometry compare immune and endocrine cellular compositions between a WT mouse without diabetes and littermate STAT3+/K392R mouse with recently-diagnosed diabetes to highlight the presence of immune infiltration with STAT3+/K392R diabetes. Results representative of viSNE plots from 8-14 week old non-diabetic WT $(n=6)$ and diabetic STAT3+/K392R $(n=4)$ mice. Error bars indicate mean +/- SD. ns = nonsignificant $(P>0.05)$, * $P £ 0.05$, ** $P £ 0.01$, *** $P £ 0.001$. 
a
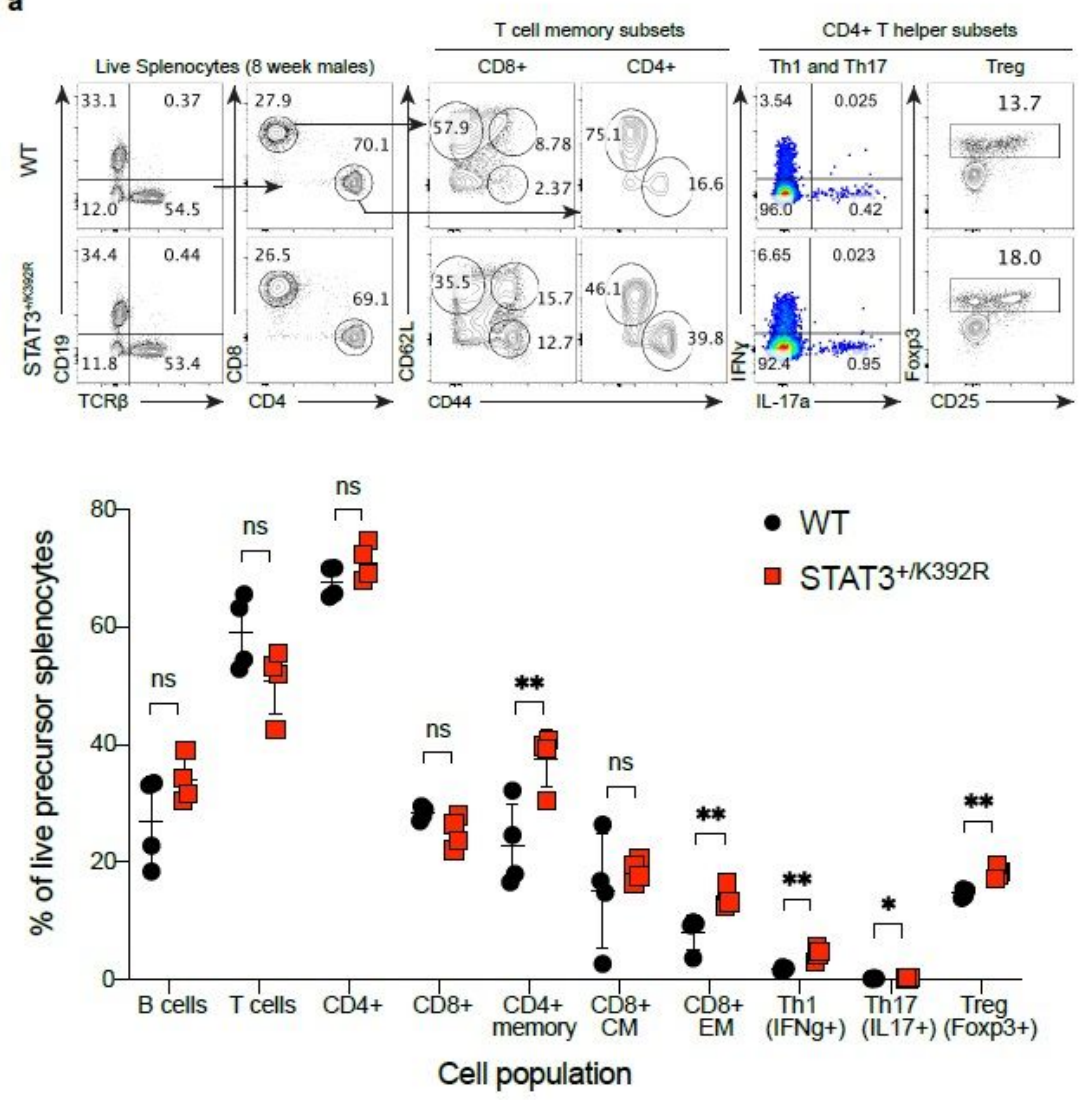

b

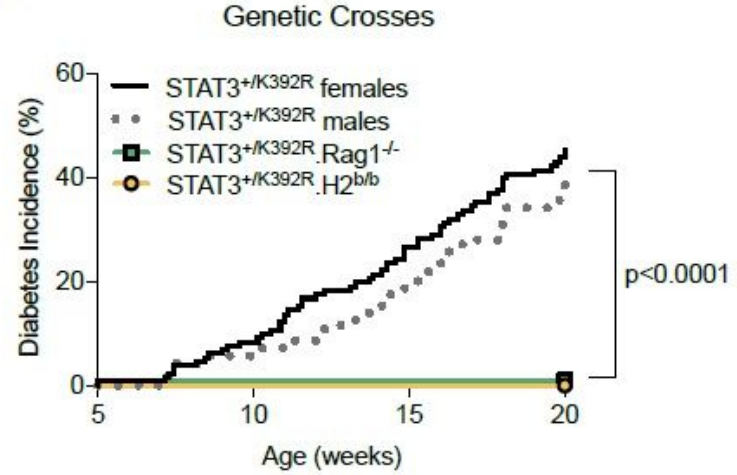

C

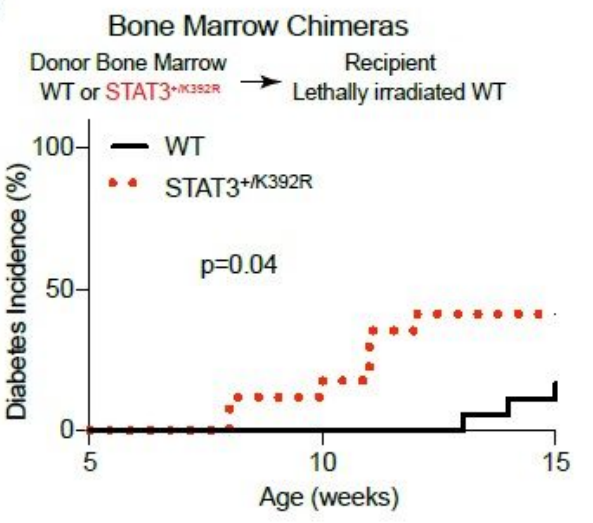

Figure 2

STAT3+/K392R effector T cell compartment | a, Immunophenotyping of splenocytes ex vivo in 8 week old male mice ( $\mathrm{n}=4$ per group) b, Diabetes incidence in STAT3+/K392R RAG1-/- mice (B- and T-cell dependence) and STAT3+/K392R H2b/b mice (MHC-dependence) (STAT3+/K392R females, n=132; STAT3+/K392R males, n=70; STAT3+/K392R RAG1-/- , n= 9; STAT3+/K392R H2b/b, n= 20) c, Experimental design and subsequent diabetes incidence in bone marrow chimeras - lethally irradiated WT recipients adoptively transferred with bone marrow from WT $(n=18)$ or STAT3+/K392R $(n=17)$ mice. Error bars indicate mean +/-SD. $n s=$ non-significant $(P>0.05)$, * $P £ 0.05, * \star P £ 0.01, * \star \star P £ 0.001$. 
a

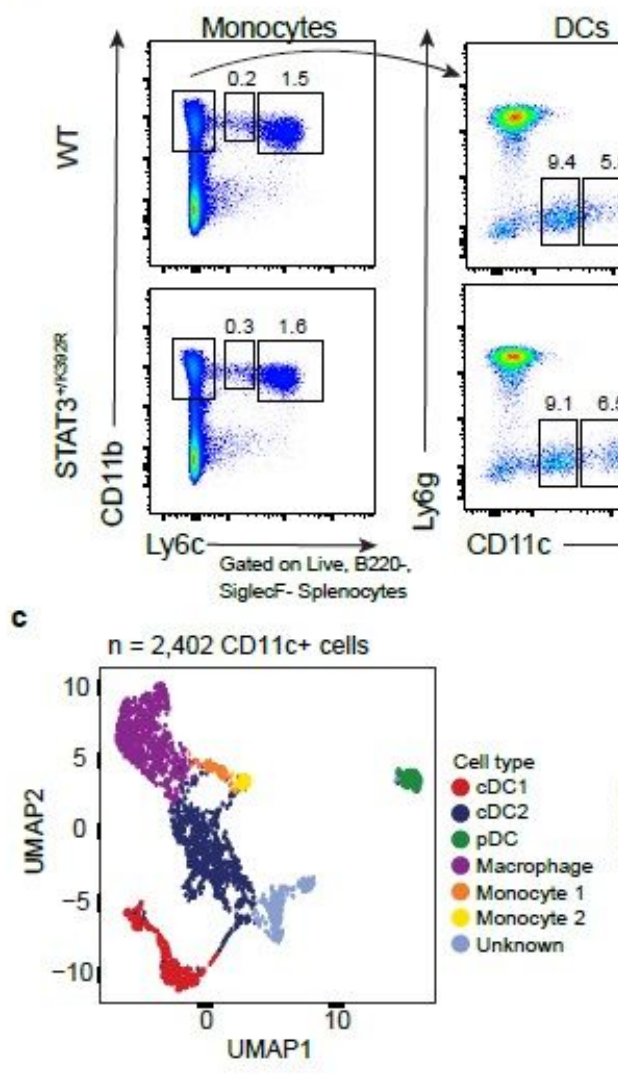

b

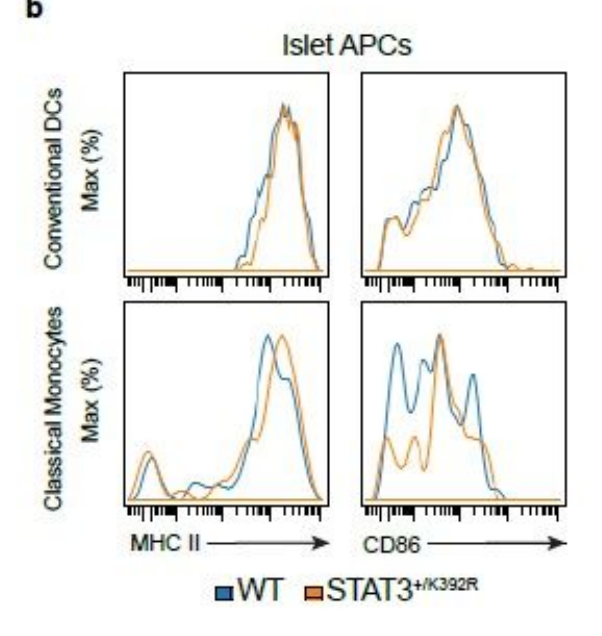

e

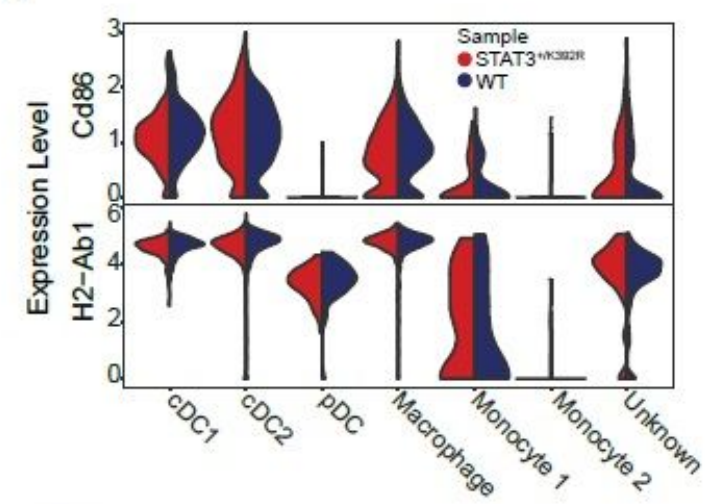

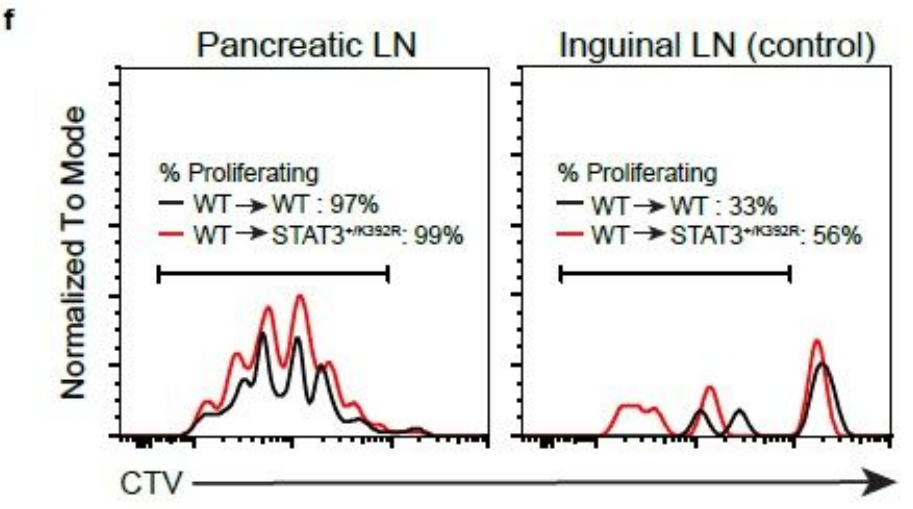
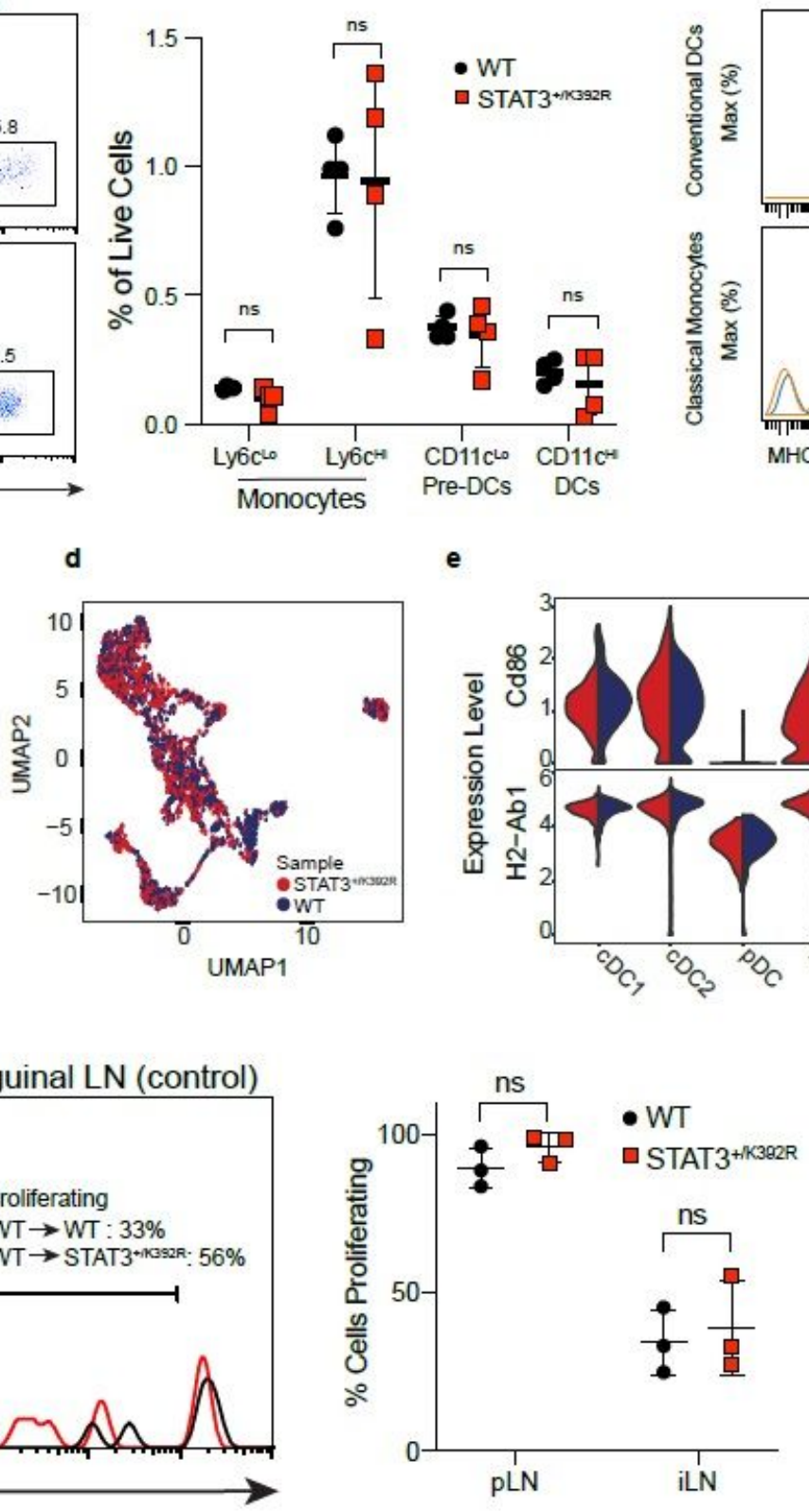

\section{Figure 3}

STAT3+/K392R APC Phenotype and T-cell priming by the local islet environment 324 is unchanged I a, Flow cytometry gating (values shown are frequency relative to parent gate) and population frequencies of APC subpopulations from splenocytes isolated ex vivo in non-diabetic 8 week old male mice $(n=4$ per group) b, Representative histograms for MHCll and CD86 protein expression on conventional DCs $(\mathrm{CD} 11 \mathrm{c}+\mathrm{MHC} \mathrm{II+)}$ and classical monocytes (CD11b+Ly6c+) in the islets of non-diabetic 8-10 week old mice using mass cytometry. Performed in 3-4 mice per group c, UMAP projection of reclustered antigenpresenting myeloid cells (CD11c+ cells) from islet immune infiltrate used in scRNA-seq experiment (see Extended Fig. 2a) d, UMAP projection showing distribution of STAT3+/K392R and WT cell transcriptomes e, Violin plots showing gene expression profile of MHCII and CD86 in APC myeloid clusters f, CTV profile 
among BDC2.5 CD4+ T cells in pancreatic and inguinal (control) lymph nodes ( $p L N$ and iLN, respectively) 3.5 days after adoptive transfer into STAT3+/K392R and WT recipients. Cell proliferation was evaluated by FACS analysis of the CTV dilution in the pLNs and iLNs of recipient mice and results are expressed as the percentage of proliferating cells within the recovered CTV-labeled BDC2.5 CD4+ T cells.

Representative of three sets of STAT3+/K392R / WT recipient mice. Error bars indicate mean +/- SD. ns = non-significant $(P>0.05)$.

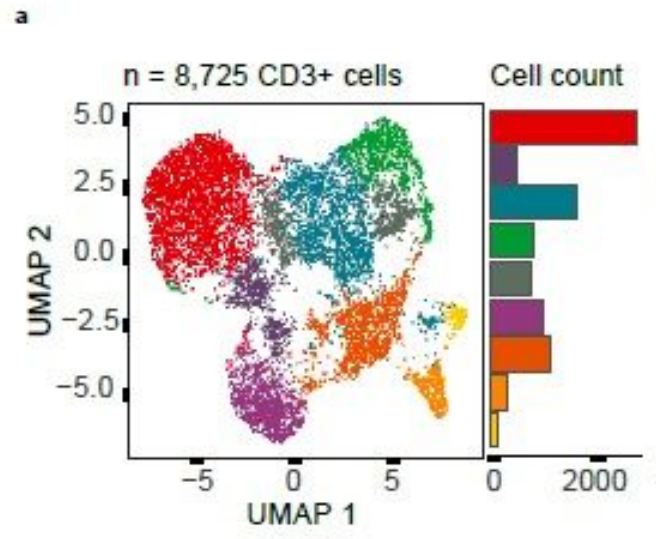

.

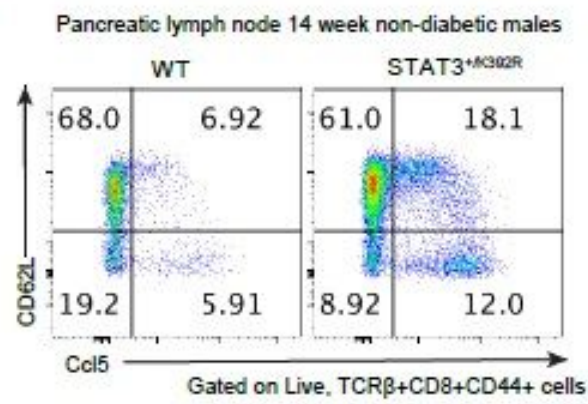

e

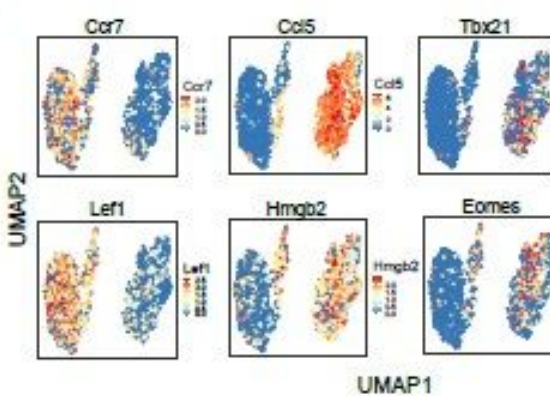

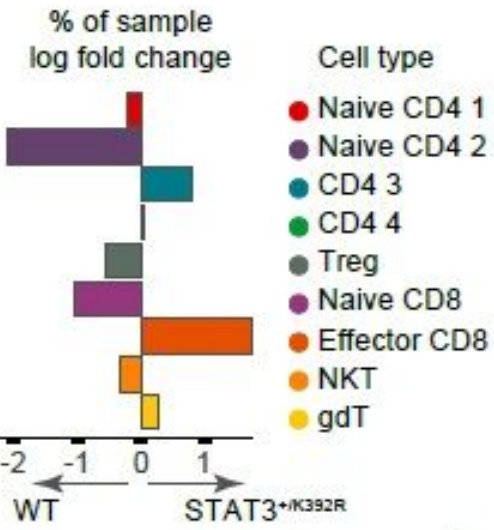

b
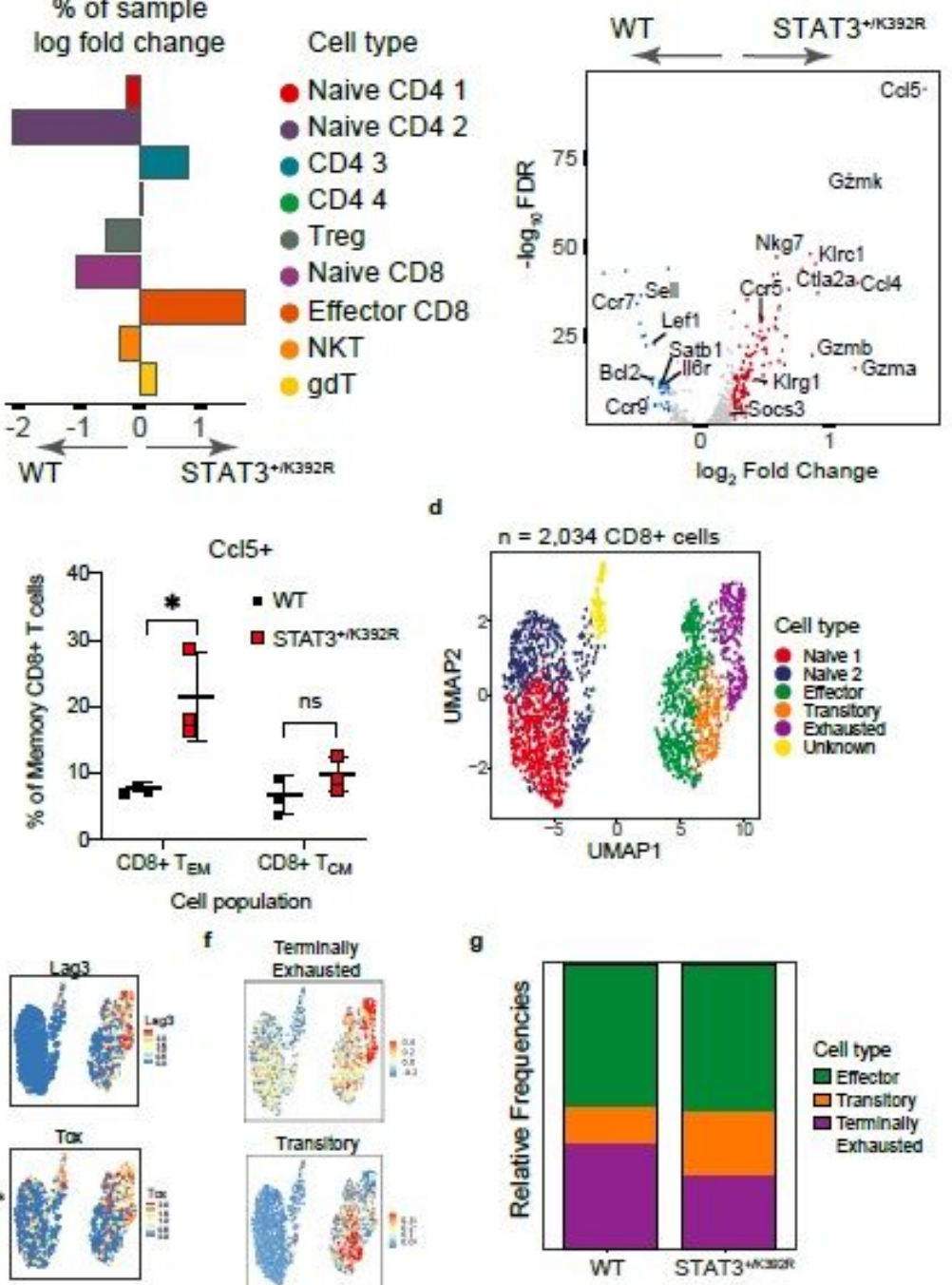

h

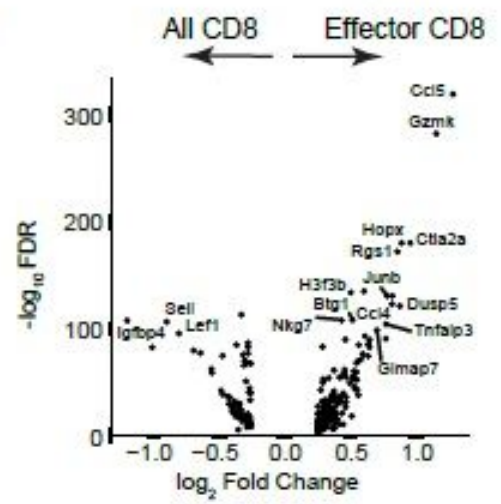

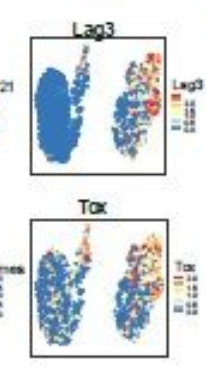
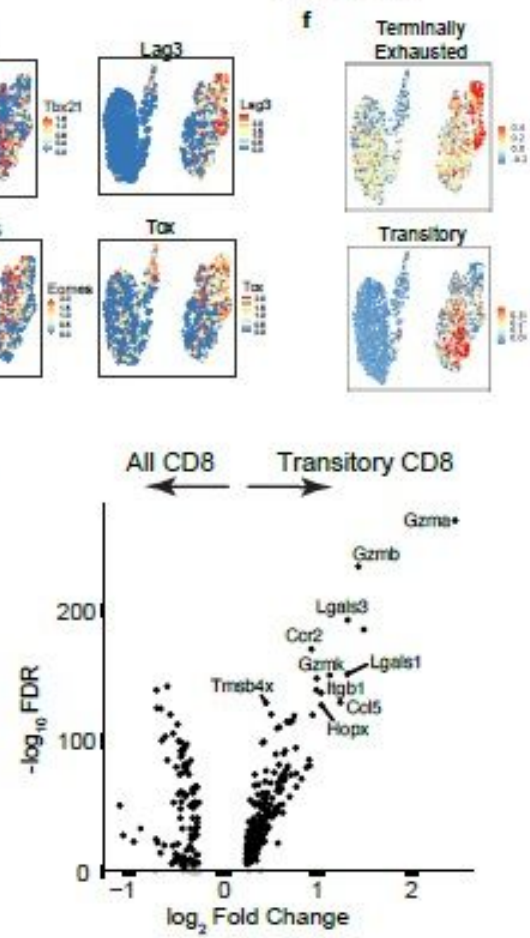
STAT3+/K392R drives expansion of the CD8+ effector compartment with upregulation of cytokine and chemokine genes | a, UMAP projection of reclustered T cells (CD3+ cells) from islet immune infiltrate used in scRNA-seq experiment (see Extended Fig. 2a) with associated cell counts per cluster and comparison of cluster frequencies between the two genotypes (STAT3+/K392R vs WT) b, Volcano plot showing STAT3+/K392R vs WT differential gene expression in CD8+ clusters. Log N fold cutoff 0.25 used. Genes of interest are labeled. c, Flow cytometry of Ccl5 protein expression in CD8+ memory T cells from the pancreatic lymph node in nondiabetic males at 14 weeks ( $n=3$ per group). Error bars indicate mean $+/-S D, n s=$ non-significant $(P>0.05),{ }^{*} P £ 0.05$. $d$, UMAP projection of reclustered CD8+ T cells e-f, Marker gene or module score expression in CD8+ $T$ cell clusters. In $\mathrm{f}$, genes used to calculate module scores for terminally exhausted T cells were Cd101, Cd200r2, Cd7, Cd200r1, II10 and for transitory T cells were $\mathrm{C} \times 3 \mathrm{cr} 1$, Klrg1, II2ra, II18rap, S1pr5, as previously described24 g, Relative population frequencies among non-naïve CD8+ cell clusters $h$, Volcano plot showing differential gene expression in CD8+ effector and transitory cell clusters relative to all CD8+ cells.

a

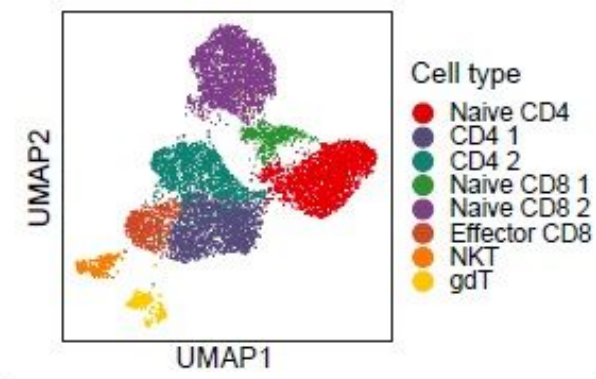

c

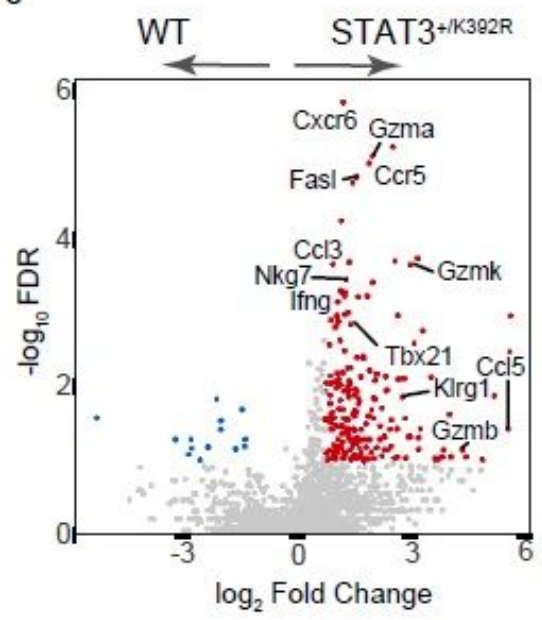

b

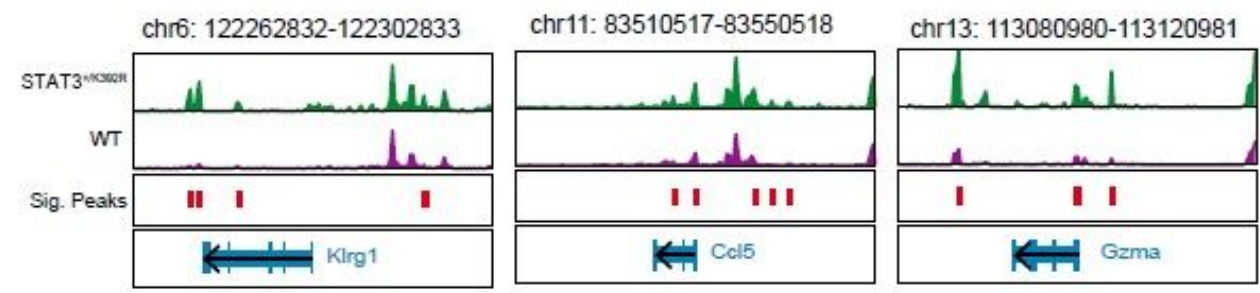

d

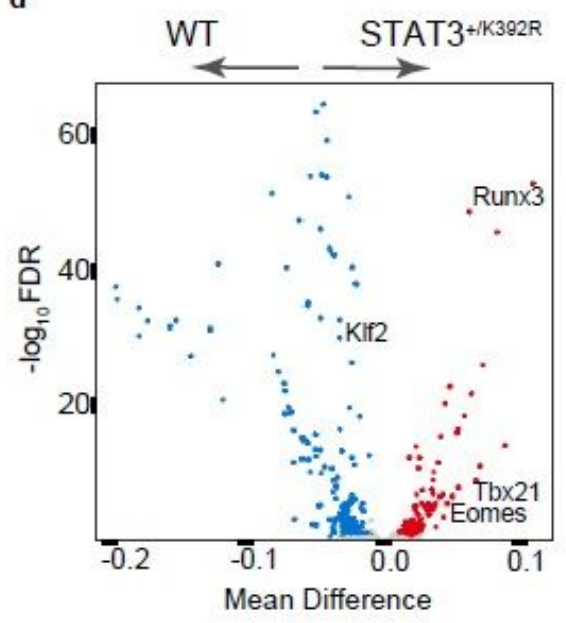

e

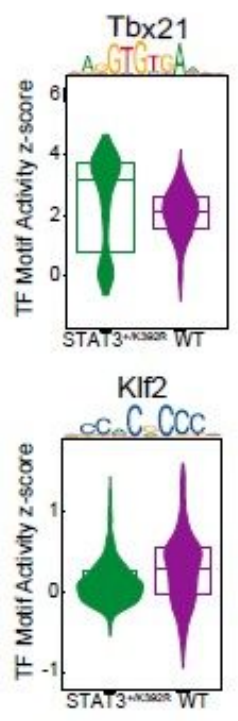

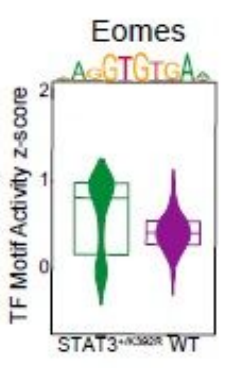

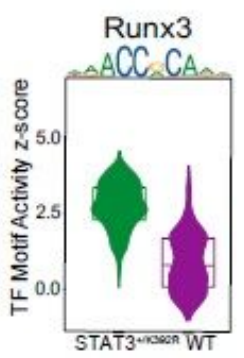

\section{Figure 5}

STAT3+/K392R increases chromatin accessibility within cytotoxic 354 CD8+ loci resulting an epigenetically distinct state | a, UMAP projection of scATAC-seq profiles of T cells (CD3+) subclustered from CD 45+ islet infiltrates of 8-10 week old male non-diabetic mice, WT ( $n=3$, pooled) vs STAT3+/K392R $(n=3$, pooled) b, Normalized pseudobulk ATAC-seq tracks of CD8+ T cell clusters split by genotype around 
genes identified as upregulated in corresponding scRNA-seq CD8+ T cell clusters. Peaks significantly upregulated (from $\mathrm{c}$ ) are shown in red c, Volcano plot showing differential peaks whose nearest gene is present in the significantly upregulated genes (Fig. 4b) between CD8+ T cell clusters (STAT3+/K392R vs WT) d, Volcano plot showing differential transcription factor motifs between CD8+ T cell clusters (STAT3+/K392R vs WT) e, Transcription factor motif activity for CD8+ T cell clusters split by genotype. Motif sequence shown above each violin plot.

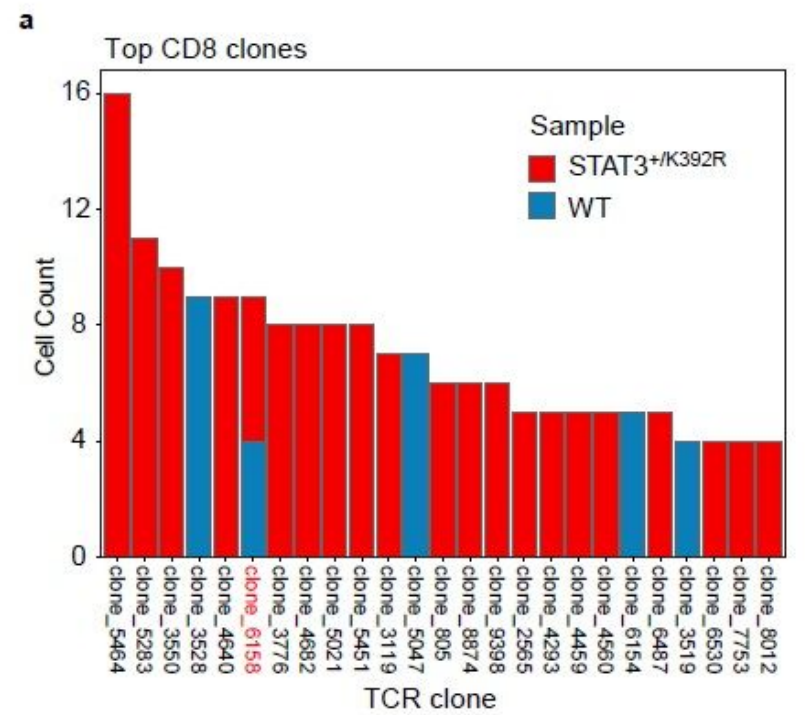

\begin{tabular}{|l|l|l|}
\hline & \multicolumn{2}{|c|}{ CDR3 } \\
\hline Clone & \multicolumn{1}{|c|}{ TCR alpha } & \multicolumn{1}{c|}{ TCR beta } \\
\hline clone_5464 & CALVDGNMGYKLTF & CASSGGQGWNSPLYF \\
\hline clone_5283 & CALSSSSFSKLVF & CASSRVDRDAEQFF \\
\hline clone_3550 & CALALRNSGGSNYKLTF & CASSFGTGGVYEQYF \\
\hline clone_3528 & CAKRNNNNRIFF & CASSFEGQNTLYF \\
\hline clone_4640 & CALSDQGGTGSKLSF & CASSLGWGSYAEQFF \\
\hline clone_6158 & CAMRDSGGSNAKLTF & CASSDSQNTLYF \\
\hline clone_3776 & CALGDRGNTGGLSGKLTF & CASSLDRGARSYEQYF \\
\hline clone_4682 & CALSDRGFASALTF & CASSLTGGRQNTLYF \\
\hline clone_5021 & CALSGPNTGKLTF & CAWSPGTGGADAEQFF \\
\hline clone_5451 & CALVAGTGGYKVVF & CASSLSDRDNSPLYF \\
\hline
\end{tabular}

\begin{tabular}{|ccc|}
\hline $\begin{array}{c}\text { TCR-8.3 } \\
\text { (IGRP-reactive) }\end{array}$ & $\begin{array}{c}\text { TCR alpha } \\
\text { CAMRDSGGSNAKLTF }\end{array}$ & $\underline{\text { TCR beta }}$ \\
CASSDAQNTLYF
\end{tabular}

c

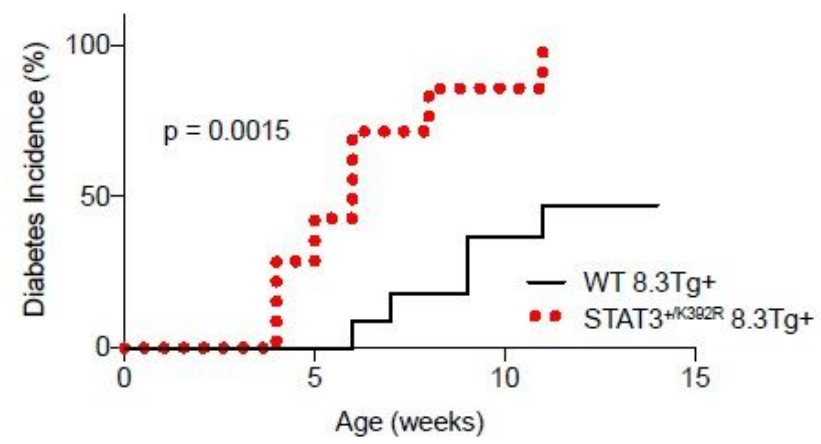

d

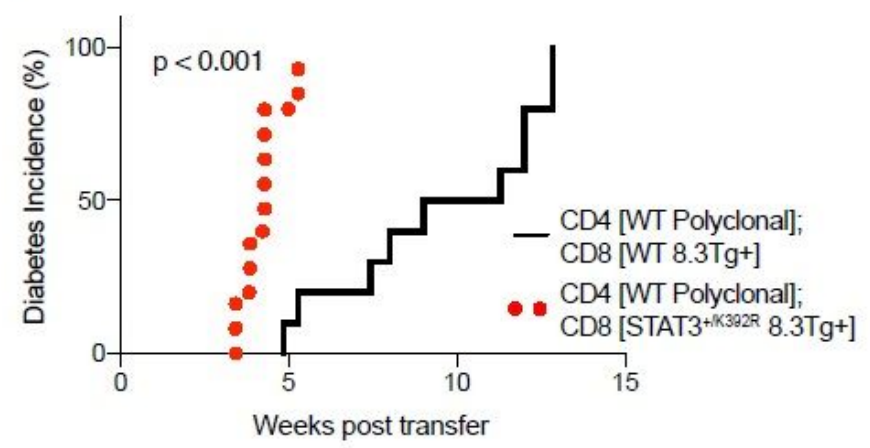

\section{Figure 6}

STAT3+/K392R drives clonal expansion of diabetogenic CD8+ T cells | a, Top 20 clones by count in each sample b, Top 10 specific clones with corresponded CDR3 sequences displayed in table. The CDR3 sequence for Clone 6158 in red is nearly identical to that of the CD8-restricted TCR specific for islet specific antigen IGRP (TCR-8.3, sequence shown below table). The only difference between Clone 6158 and TCR- 8.3 is a serine, denoted in blue, replaces an alanine of the TCR beta chain c, Diabetes incidence of 8.3Tg+ (IGRP-specific TCR) mice with $(n=7)$ and without $(n=11)$ STAT3+/K392R confirms increased diabetogenicity d, Diabetes incidence after adoptive transfer of polyclonal WT CD4+ T cells with naïve CD8+ T cells from 8.3Tg+ mice with $(n=5)$ and without STAT3+/K392R $(n=10)$ into NOD.SCID mice. Results are pooled from 2 independent cohorts. 